\title{
Quantifying the contribution of occupational segregation to racial disparities in health: A gap-closing perspective *
}

\author{
Ian Lundberg ${ }^{\dagger}$
}

July 3,2021

Word count: 8,728

Keywords: health disparities, occupational safety, causal inference, quantitative methods

\begin{abstract}
Racism causes racial disparities in health, and structural racism has many components. Focusing on one of those components, this paper addresses occupational segregation. I document high onset of work-limiting disabilities in occupations where many workers identify as non-Hispanic Black or as Hispanic. I then pivot to a causal question. Suppose we took a sample from the population and reassigned their occupations to be a function of education alone. To what degree would health disparities narrow for that sample? Using observational data, I estimate that the disparity between non-Hispanic Black and white workers would narrow by one-third. This estimate is credible because of adjustment for lagged measures of demographics, human capital, and health carried out under transparent causal assumptions. The result contributes to understanding about inequality and health by quantifying the contribution of occupational segregation to a disparity: if we took a sample and reassigned occupations, the disparity would narrow but would not disappear. The paper contributes to methodology by illustrating an approach to macro-level claims (how segregation affects a population disparity) that draws on explicitly causal micro-level analyses (potential outcomes for individuals) for which data are abundant.
\end{abstract}

${ }^{*}$ A draft of this working paper is available on SocArxiv [link]. Replication code is available on GitHub [link] and will be posted on Dataverse when the paper is forthcoming. For helpful discussions and feedback relevant to this project, I thank Brandon Stewart, Matthew Salganik, Rebecca Johnson, Gillian Slee, and Dalton Conley. Research reported in this publication was supported by The Eunice Kennedy Shriver National Institute of Child Health \& Human Development of the National Institutes of Health under Award Number P2CHD047879.

${ }^{\dagger}$ Ph.D. Candidate, Department of Sociology and Office of Population Research, Princeton University, ianlundberg.org, ilundberg@princeton.edu, 227 Wallace Hall, Princeton University, Princeton, NJ 08540. 


\section{Introduction}

From 1930-1932, roughly 5,000 men drilled a tunnel through Gauley Mountain in West Virginia. The drills dispersed clouds of silica dust through the air and into the lungs of the workers, who were given no protective equipment. At least 764 workers died of silicosis, a serious lung condition. The Hawk's Nest Tunnel has been called the worst industrial disaster in American history (Cherniack, 1986). Further, the roughly 5,000 men employed to dig the tunnel were not a random cross-section of society: two-thirds were Black. The tragedy of the Hawk's Nest Tunnel was not only the failure to use protective equipment; it was also the disproportionate allocation of Black workers into a job with high risk.

Disparate placement into dangerous occupations occurs in smaller form throughout the U.S. economy (Fig 1). Home health aides endure risk to physically support others; $31 \%$ identify as non-Hispanic Black. Agricultural workers face hazards to produce the food we consume; 46\% identify as Hispanic. From industrial truck operators (19\% Black, 21\% Hispanic) to personal care aides (19\% Black, 17\% Hispanic) to roofers (5\% Black, 41\% Hispanic), people of color hold occupations with health hazards. In fact, the placement of people of color in harm's way has been part of the American economy since the time of slavery.

This paper contributes a new perspective on inequality and health by examining the degree to which occupational segregation contributes to racial health disparities in the onset of work-limiting disabilities. It may seem uncontroversial to say that occupational segregation plays an important role, but it is not the only force. Health disparities are also the product of experiences of racism, residential segregation, access to healthy food, and many other factors. The degree to which one specific input-occupational segregation - is responsible for health disparities is an open topic for research. The magnitude matters: if occupational segregation accounts for $20 \%$ versus $80 \%$ of the disparity, those would point to different areas for future research and different implications for what types of policies could help to close health disparities.

The paper also contributes to quantitative methodology by illustrating one approach 
A) Occupations arranged by proportion non-Hispanic Black

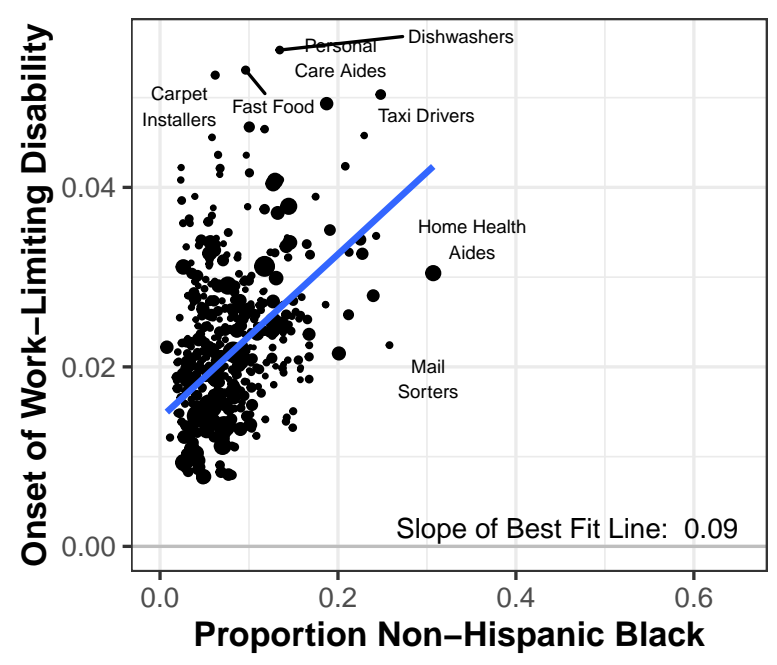

C) Occupations arranged by proportion Hispanic

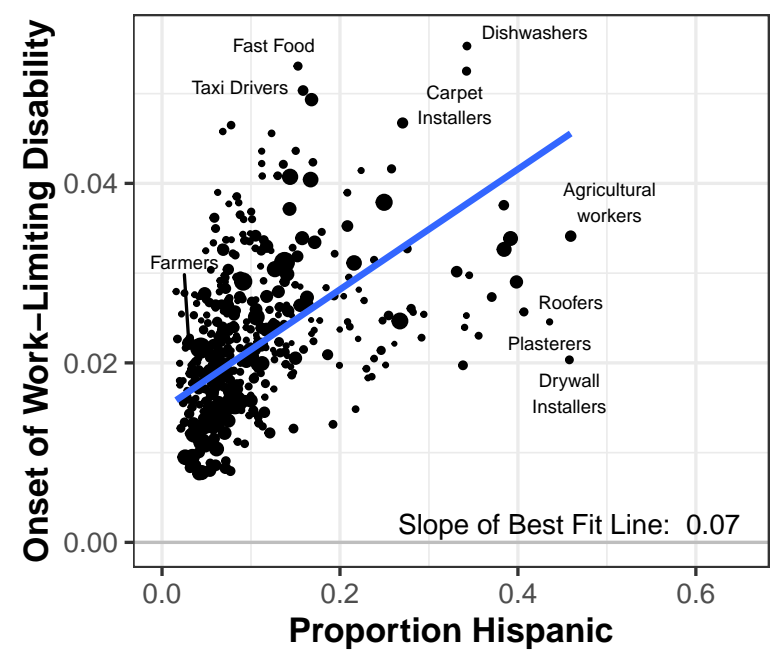

B) Occupations arranged by proportion non-Hispanic white

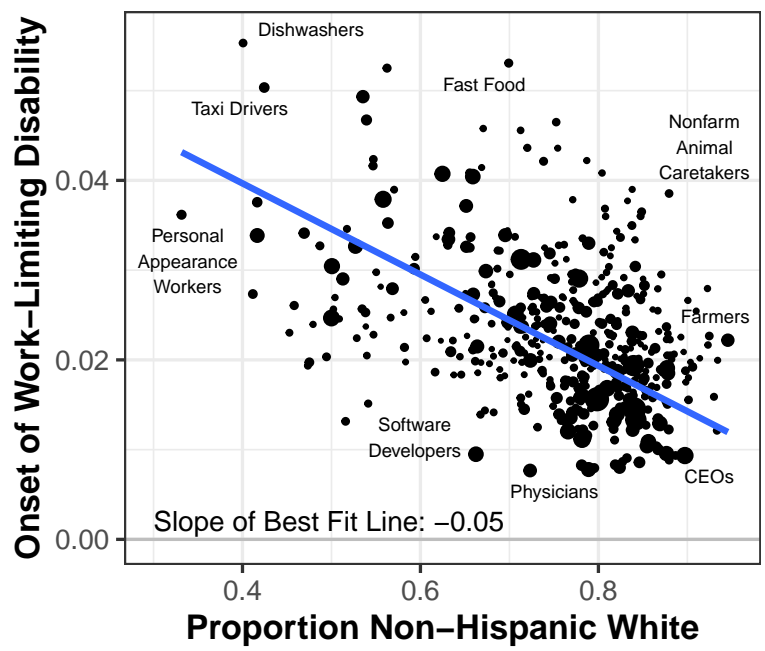

D) Occupations arranged by proportion of other categories

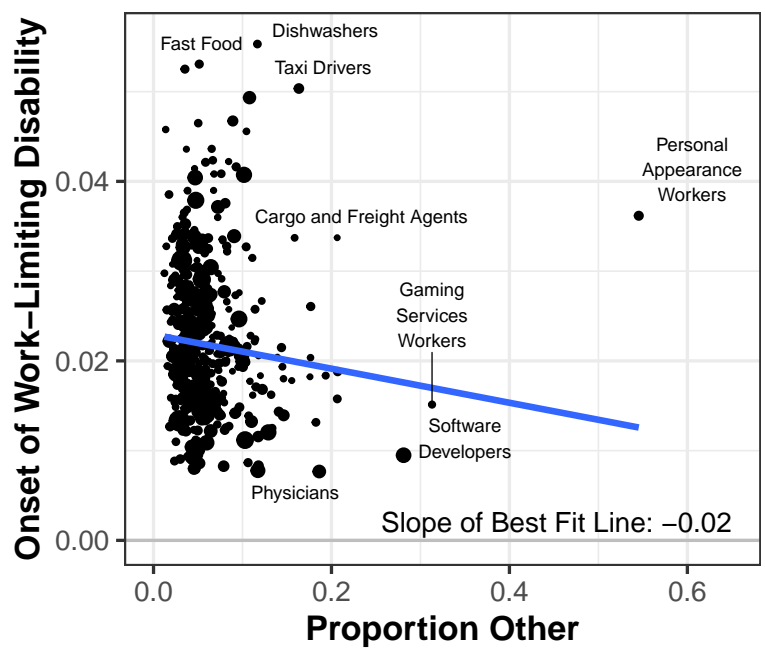

Fig. 1. Dangerous occupations are disproportionately filled by people of color. Each point is an occupation scaled proportional to its size in the full population. The horizontal dimension is an estimate of the proportion identifying with a given racial and ethnic category in the occupation in year $t$. The vertical dimension is the proportion of all people in that occupation for whom a disability or health condition limiting the kind or amount of work they can do is reported in year $t+1$. Both the horizontal and vertical dimensions are estimated by multilevel models with random intercepts using data from the 2005-2020 Annual Social and Economic Supplement of the Current Population Survey (details in Appendix B). 
for linking micro-level evidence to macro-level claims. Micro-level evidence is abundant: we can observe many people in many occupations, with disparate health outcomes. But sociological interest may lie in the macro-level implications of those micro-level outcomes: how occupational segregation (at the population level) contributes to a health disparity (at the population level). This paper presents one approach for making that connection. Each individual $i$ has a potential health outcome $y_{i}(d)$ that they would realize if exposed to each possible occupation $d$. They also have an expected outcome $\bar{y}_{i}(\pi)$ realized if they were counterfactually assigned to an occupation from an equitable assignment rule $\pi$. Aggregating over individuals, we can return to the macro level to estimate a counterfactual population health disparity. The cost of a micro-level approach is that the result should be interpreted as the outcome of a hypothetical experiment: if we drew a sample from the population and desegregated occupations for that sample, what is the expected health disparity we would observe in the sample? This procedure draws on recent methodological advances to place racial disparities in a causal perspective (Jackson and VanderWeele, 2018; Lundberg, 2021; VanderWeele and Robinson, 2014).

The paper proceeds in several sections. Section 2 argues that occupational segregation contributes to health disparities but is not the whole story: the contribution is likely to be much more than $0 \%$ but much less than 100\%. Section 3 introduces the analytical framework for linking micro-level evidence to a macro-level claim. Section 4 present the data, causal assumptions, and estimation methods. Section 5 shows that occupational segregation is partially responsible for the Black-white disparity in reported onset of work-limiting disabilities, accounting for $36 \%$ of the disparity. Section 6 concludes by discussing implications for inequality and health as well as for quantitative methodology. 


\section{Occupational segregation likely contributes to health disparities but is not the whole story}

Occupational segregation has been deeply intertwined with racial inequality throughout American history. In the colonial and antebellum periods, white traders reaped profit by forcing African people into bondage and transporting them to America to fill an exploitative labor market role in Southern agriculture. The Civil War brought legal emancipation, yet short-lived Reconstruction-era policies did little to produce economic opportunity. Sharecropping became pervasive in the South, and even in Northern cities economic barriers maintained racial inequality (Du Bois, 1899). Nor did the rapid industrialization of the early 20th century eliminate occupational segregation, as illustrated by the Hawk's Nest Tunnel (1930-1932) from the opening of this paper.

Against this historical backdrop, the Civil Rights Act of 1964 proclaimed an end to hiring discrimination on the basis of race. Much of the sociological literature on occupational segregation begins the historical journey at that point. Examining racial segregation across nine occupational categories (e.g. service workers) recorded by the Equal Employment Opportunity Commission (EEOC), past studies document only a partial integration of occupations since 1964 (Ferguson and Koning, 2018; Stainback and Tomaskovic-Devey, 2012; Tomaskovic-Devey et al., 2006). The period after the Civil Rights Act thus continued the same pattern that had already characterized the long run of American history: movements toward occupational integration that were incomplete and faltering. But to what degree does incomplete integration contribute to health disparities?

\subsection{Much more than 0\%: Occupational segregation shapes health}

In 2019, 2.8 million Americans experienced workplace injuries (Bureau of Labor Statistics, 2020). Compared with white workers, Black workers disproportionately hold occupations with high injury rates (Seabury et al., 2017; Stanbury and Rosenman, 2014). Case studies 
reveal specific links between particular occupational hazards and health outcomes, from heat-related illness among farmworkers (Fleischer et al., 2013) to physical trauma among day laborers (Lowry et al., 2010) and hotel workers (Buchanan et al., 2010). Occupations also expose individuals to unequal toxins with effects that may accumulative over time; for example, Juon et al. (2021) find that Black workers hold occupations that place them at higher exposure to dangers such as silica and asbestos, which increase the risk of lung cancer.

The total contribution of the work environment to health is likely to be even greater than those studies suggest because direct injuries and exposures are only a small slice of all the links between work and health. For example, random assignment to a work environment that allowed flexible hours increased sleep and exercise among workers in one corporate case study (Moen et al., 2011). In the opposite direction, workers in precarious employment situations with constantly shifting work schedules report lower quality sleep (Harknett et al., 2020). Work characteristics are related to health behaviors like alcohol consumption (Prins et al., 2019). An exclusive focus on injuries and illnesses tied to specific workplace experiences would miss these indirect consequences. Scholars have therefore called for a more comprehensive research agenda including both direct and indirect links between work and health (Ahonen et al., 2018; Burgard and Lin, 2013; Lipscomb et al., 2006; Schulte et al., 2017). In response to that call, it is important to study the effect of occupations as a whole rather than focusing solely on specific occupational hazards.

\subsection{Much less than 100\%: Structural racism is multifaceted}

But occupational segregation is not the only input that produces racial health disparities; structural racism has many components (Williams et al., 2019). The inputs to racial health disparities range from exposures to environmental contaminants at home (Banzhaf et al., 2019) to experiences of racism in everyday life (Lewis et al., 2015; Williams and Mohammed, 2009). An extensive literature has documented how these stressors get under the skin in a 
cumulative process of weathering (Geronimus, 1992) that creates a physiological stress response measurable in indicators such as elevated blood pressure and cholesterol (Geronimus et al., 2006; McEwen, 1998; Merkin et al., 2009). Even if we were able to eliminate occupational segregation, that would not eliminate the plethora of other sources that would still produce racial health disparities. For this reason, we should not expect that occupational segregation would fully account for the magnitude of health disparities.

\subsection{Quantifying the magnitude: An open question}

Occupational segregation is likely responsible for substantially more than $0 \%$ but substantially less than $100 \%$ of the magnitude of racial health disparities. But it is difficult a priori to predict precisely how important it is. Even conceptualizing that question is somewhat difficult: what does it mean to say that segregation contributes to a disparity? The next section advances one possible definition: occupational segregation contributes in the sense that the disparity would be different under some hypothetical intervention to desegregate occupations.

\section{Analytical framework: Bringing micro-level evidence to bear on macro-level claims}

Occupational segregation can be conceptualized as a macro-level phenomenon: the unit of analysis would be the U.S. population, for which occupational segregation takes some particular value. A common approach to macro-level questions is to examine variation across social contexts such as neighborhoods, states, or countries (Heymann, 2003; Krieger, 2001; Marmot and Wilkinson, 2005; Ólafsdóttir and Beckfield, 2020). For example, TorratsEspinosa (2021) follows that strategy to estimate the causal effect of residential segregation (defined at the county level) on racial disparities in COVID-19 mortality (defined at the

county level), net of control variables (defined at the county level). That works because 
one can observe many counties - the entire analysis is at the macro level. But for some theoretical questions there is only one macro-level context. In the present case, there is only one U.S. society. Empirical considerations in this setting therefore require that the macro-level question be linked to a micro-level analysis for which the unit of analysis is a person.

A long theoretical tradition has focused on the linkage between micro and macro. Much of this tradition has called for (1) theories about how individual actors select actions and (2) theories about how those actions interact to produce collective phenomena (Coleman, 1990). For example, Bearman et al. (2004) theorize rules by which people select romantic ties and show by simulation that those rules aggregate up to network patterns similar to those observed in reality. The subfield of analytical sociology has most explicitly carried forth a theoretical aspiration toward "grounding the macro in the purposeful actions of individuals," (Hedström and Bearman 2009:37).

But sometimes our social science knowledge is inadequate to theorize the behavior of actors or how they interact with each other. In this setting, we can still do something useful. This paper defines causal effects at the individual level (without theorizing how they derive from specific actor behaviors) and aggregates those to a population-level claim (without addressing interactions among units). The cost of this more limited claim is that it does not address situations where the whole may be more complex than the sum of the parts. But, there are settings in which the result is still useful for science and for policy. In the absence of strong theory about the interactions among units that would make the whole different from the sum of the parts, an estimate that focuses on the sum of the parts is an important first step. The analytical framework proceeds in three steps: (1) define potential outcomes for individuals, (2) theorize an equitable treatment assignment rule, and (3) interpret results in terms of the initial response to a policy change. 


\subsection{Define potential outcomes for individuals}

Suppose each person $i$ has a potential outcome $y_{i}(d)$ : the health (healthy or with a worklimiting disability) that they would report if assigned to occupation $d$ (Imbens and Rubin, 2015). Because there are 451 occupations in the Census coding system, that corresponds to 451 potential outcomes for each person. ${ }^{1}$ Yet we observe only one: the outcome in the occupation they factually held. Nonetheless, defining all of the potential outcomes allows us to move beyond descriptive disparities and toward disparities in a counterfactual world with occupations allocated differently, under a hypothetical intervention to change the occupation to which each individual is assigned.

To illustrate, suppose we have a hypothetical population of eight people assigned to one of three occupations (Fig 2). We are interested in the health disparity across two categories of people, here labeled abstractly as circles and diamonds but which could correspond to categories of race, class, or gender. In this hypothetical setting, Panel A shows a health disparity: only $\frac{1}{4}$ of the circles will develop a disability next year compared with $\frac{3}{4}$ of the diamonds. That disparity could be a consequence of segregation: the diamonds are disproportionately home health aids, an occupation which (in this hypothetical example) is very hazardous. What if each person was instead assigned to a random occupation, so that the disparity in that counterfactual world could not be attributed to occupational segregation? If we knew the full table of potential outcomes (Panel B), we could answer that question: randomly assigning occupations would reduce the disparity from $\frac{1}{2}$ to $\frac{1}{4}$. That provides a metric of the degree to which occupational segregation contributes to a disparity. One can extend this idea to more complex counterfactual rules for assigning occupations (next subsection) and must make causal assumptions in order to learn about unobserved potential outcomes (next section). ${ }^{2}$

\footnotetext{
${ }^{1}$ The empirical analysis focuses on the 428 occupations for which the data contain people of all four racial categories studied.

${ }^{2}$ The treatment here is occupation: we are imagining that each person could counterfactually have held a different occupation. This operationalization stands in contrast with a common approach in existing work: scaling occupations to a continuous score. For example, Fujishiro et al. (2017) estimate the degree
} 
The contribution of occupational segregation can be theorized as the difference between a factual and a counterfactual disparity

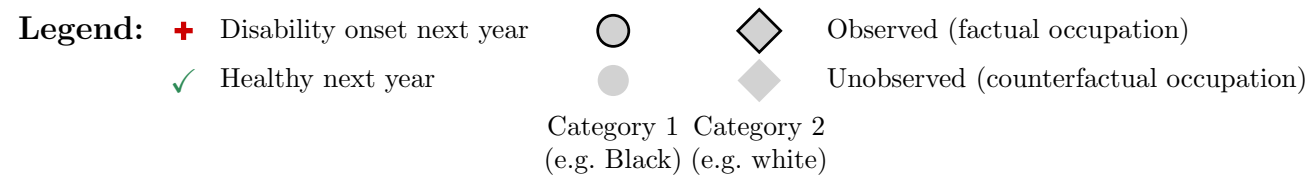

A) Factual disparity with occupational segregation as observed

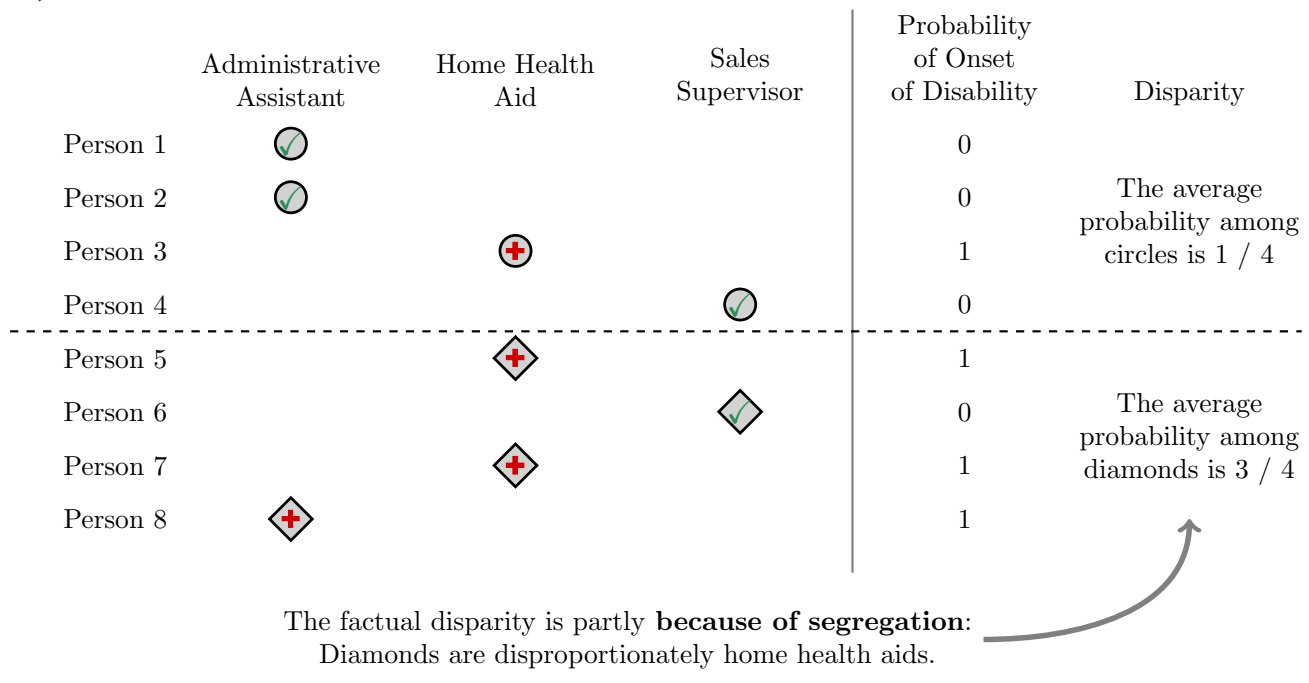

B) Counterfactual disparity if occupations were assigned at random

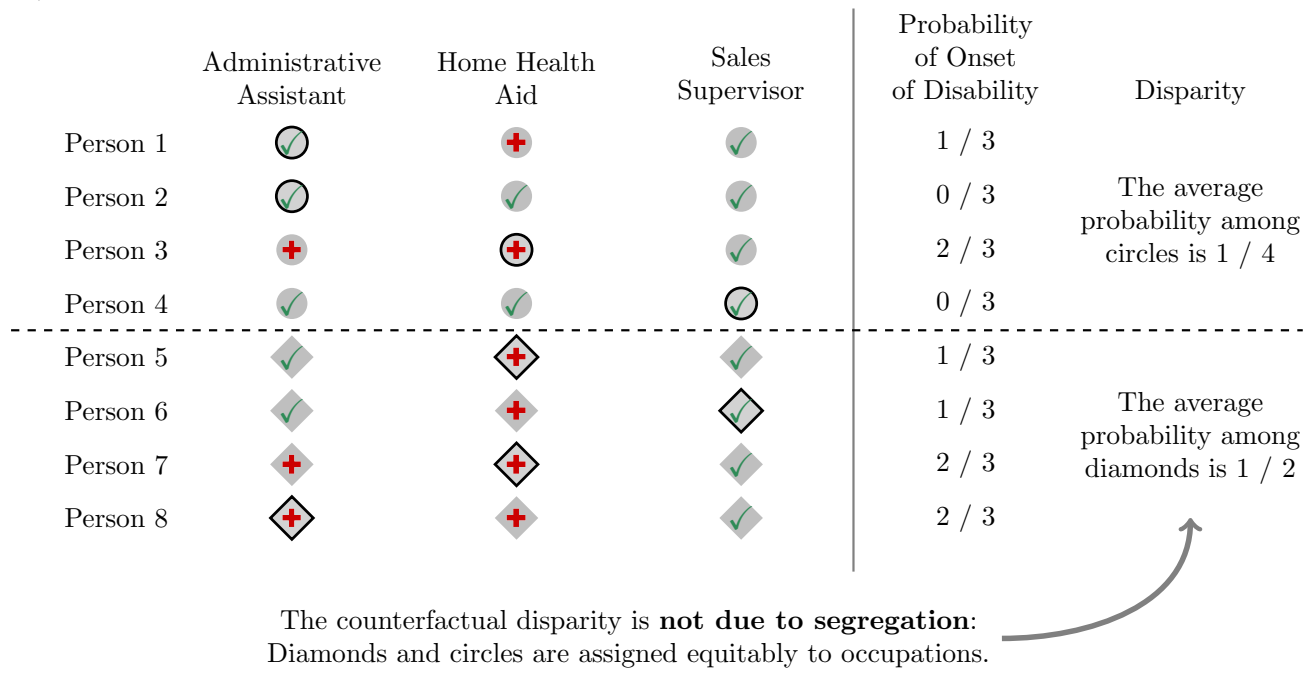

\section{Fig. 2. Theoretical framework for consequences of occupational segregation.}

This conceptual figure considers a hypothetical population of eight people assigned to three possible occupations. Each person has a factual occupation in which they either will or will not develop a work-limiting disability next year (Panel A). The factual health disparity is partly a consequence of occupational segregation. Suppose we knew the health outcome each person would realize if they had counterfactually held any occupation (Panel B). For example, Person 1 would develop a disability only if they were a home health aid. If we knew these counterfactual outcomes, we could calculate the disparity that would persist if occupations were assigned at random. The difference between the factual and counterfactual disparities would then correspond to the contribution of occupational segregation to the health disparity. Section 4 presents the assumptions and methods required to support that type of theoretical claim. 
Importantly, this approach makes no appeal to change a circle into a diamond or vice versa; the causal effect of the category (circles and diamonds) remains undefined. Rather, circles and diamonds are simply viewed as labels that distinguish two subpopulations of people. That perspective is enormously helpful when the disparity is defined over a category like race. Because it is difficult to conceptualize an intervention to change someone's race, statisticians refer to race as an "immutable" characteristic for which causal effects are undefined (Greiner and Rubin, 2011; Holland, 1986). You might be able to manipulate one component like the name at the top of a resume (Bertrand and Mullainathan, 2004; Sen and Wasow, 2016), but some have proposed that doing so is too limiting (Kohler-Hausmann, 2018) because it reduces a socially and historically constructed category (Omi and Winant, 2014) to one signal. In short, any statement about the outcome a given person would realize if they were white versus if they were Black leads into heavily contested questions about what it means to be white or Black. The approach taken in this paper avoids that problem by defining counterfactuals over occupations, thus only requiring an answer to questions like what it would mean for a given person to be a home health aid or a sales supervisor. We get to maintain race in all its complexity. This pivot appears in epidemiology (Jackson, 2021; Jackson and VanderWeele, 2018; VanderWeele and Robinson, 2014) and sociological methodology (Lundberg, 2021), and the present paper applies those ideas in the specific setting of occupational segregation.

\subsection{Theorize an equitable assignment rule}

The illustration above proposed assigning people to occupations at random. To define a more complex assignment rule, a helpful abstraction is to consider occupations as though

to which racial disparities in mortality are attributable to the causal effect of the substantive complexity of one's occupation (a scale involving the types of reasoning tasks done on the job). The scaling approach may seem more general, since one might imagine an intervention to change the tasks carried out in an occupation without changing the assignment of people to occupations. But in practice when a continuous scale is a deterministic function of occupational categories, there is no way to change someone's value on the continuous scale without changing their occupation. This is why it is helpful to think about the treatment as the occupation itself, rather than a score to summarize the occupation. 
they were assigned by lotteries (Fig 3). In the world as it exists, each person has their own lottery: the probability that they hold any given occupation is a function of all of their characteristics, some of which might be deemed fair (e.g. education) and some of which might be deemed forms of unfair discrimination (e.g. race). When designing an equitable counterfactual lottery, we get to specify the counterfactual probability of assigning each occupation $d$ to each person $i$ such that the probability is only a function of the former, which Jackson (2021) terms allowable covariates. In this case, we can imagine counterfactually shuffling the assignment of people to occupations within subpopulations defined by education.

This counterfactual lottery is particularly helpful because educational credentials constrain access to occupations (Blau and Duncan, 1967; Hout, 2012). Those without a college degree are not able to be counselors or social workers, for example. A counterfactual that shuffles occupations within educational categories rather than in the population as a whole avoids unrealistic counterfactuals such as assigning a person with less than a high school degree to be a counselor. Eq 3.1 formalizes this equitable assignment rule mathematically as a function $\pi()$ that takes as inputs an occupation $d$ and a value of education (less than high school, high school, some college, college) and returns a counterfactual probability of assignment to occupation $d$ given that education category. ${ }^{3}$

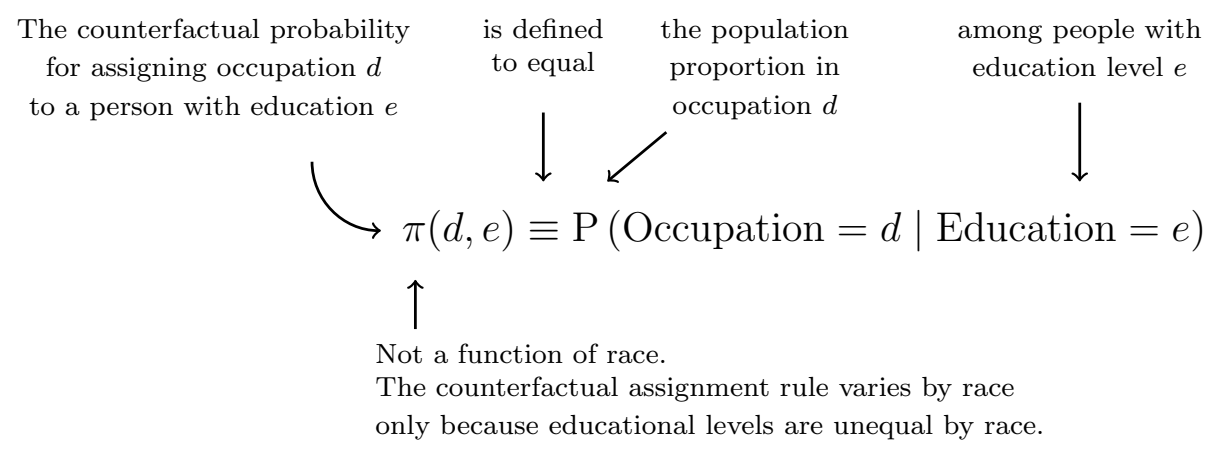

Importantly, the equitable assignment rule above does not fully equalize occupations:

\footnotetext{
${ }^{3}$ The choice to define the lotteries by these four educational categories is a conceptual rather than a data-driven choice. One could use a more granular definition of education or include other covariates in the assignment rule. These four educational categories are chosen as a parsimonious definition of categories within which all observed occupations are conceptually realistic for any given individual.
} 


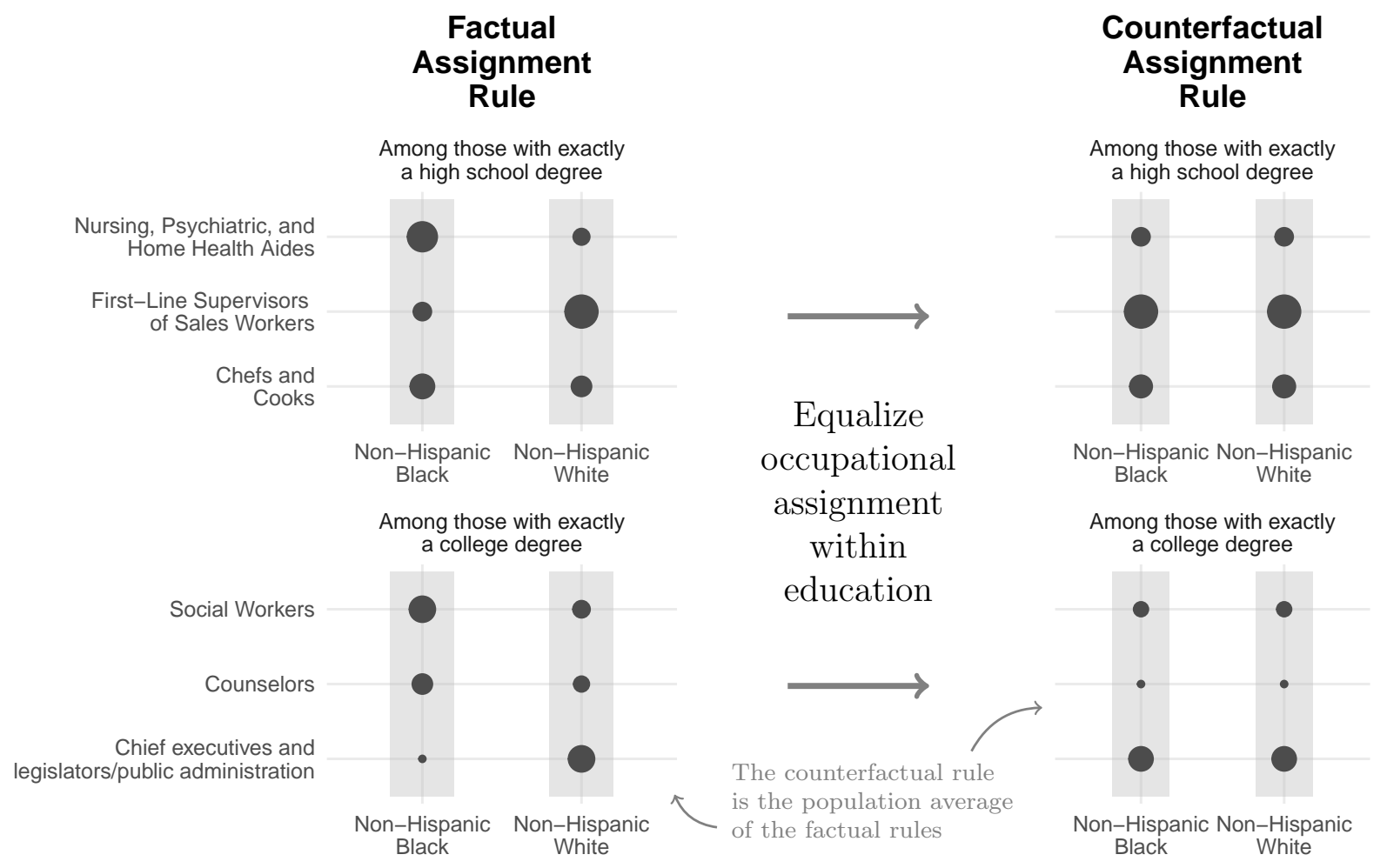

Fig. 3. A counterfactual assignment rule illustrated with selected occupations. Each gray box corresponds to a lottery from which an individual is assigned an occupation. Circle sizes represent the probability of that particular occupation. The factual assignment rule at the left is segregated and inequitable: the probability of receiving an occupation is a function of race even within educational categories. The counterfactual rule at the right is equitable: the probability of assignment to an occupation is only a function of one's education. The empirical analysis to follow carries out this exercise with occupational categories counterfactually assigned by probabilities defined within four levels of education (less than high school, high school, some college, college). The analysis restricts to the 428 occupations in which people of all four racial categories are observed. 
the probability of assignment to an occupation remains a function of race indirectly because education is a function of race. But this rule allows us to focus on one step of the stratification process - occupational assignment given education - and leaves to future work questions about how educational inequality contributes to health disparities.

\subsection{Study the initial response to a policy change}

There is a cost to taking a micro-level approach to a macro-level question. A focus on individuals lends itself to claims about individuals - what would happen to a given person if they held a different occupation? It becomes much harder to make claims about populations - what would happen if everyone held a different occupation? The latter question faces problems because changing the occupation of one person might affect another person's outcome. Purely macro-level analyses (comparisons across countries with different

levels of occupational segregation) would avoid this problem by taking the entire country as the unit of analysis, but they also come with drawbacks: they cannot be used to make claims specific to the U.S., they are difficult when race means something different across countries, and they render it difficult to ask questions about counterfactuals with integrated occupations because no country has integrated occupations. It is therefore worthwhile to find an interpretation of the micro-level analysis that avoids some of the interference problems that inhibit its ability to speak to the full population.

Lundberg (2021) proposes a solution that appeals to hypothetical samples: suppose we took a small sample from the population and reassigned occupations for that sample. What disparity would remain in the sample? That procedure avoids many interference problems because we are only intervening on a fraction of the population, who may not interact with each other. The target of inference is then the expected disparity across all hypothetical samples from the population. The sampling-based target is relevant to policy: when creating a new policy, we can at best apply that policy to only a fraction of the population at first. For instance, it is easier to think about a policy to move one Black worker out of the Hawk's 
Nest Tunnel by helping that worker find a different job. It is harder to think about a policy to change the entire composition of the Hawk's Nest workforce. A local target also lends itself to continued updating of the evidence: after applying the policy to some fraction of the population, we could re-estimate the gap-closing estimand before applying it to another sample. A micro-level analysis of a macro-level claim cannot tell us what would happen if everyone's occupation changed at once, but it can tell us the initial response to a policy change.

Carefully scoped interpretations are especially important for racial health disparities, for which population-level interventions could easily change the structure of the problem. Suppose thousands of white workers were counterfactually assigned to dig the Hawk's Nest Tunnel instead of thousands of Black workers. In that case, racist supervisors might have felt compelled to distribute protective equipment. In the language of Jackson (2021), that intervention is not system preserving: it not only changes the distribution of the treatment (occupation), but also changes the mapping between treatments and outcomes (how dangerous the occupation is). In health disparities, many population-level interventions are not system preserving because those with resources find new ways to maintain their advantages (Link and Phelan, 1995). This makes it all the more important to address interference problems head-on, either by encoding interactions in a formal model (Jackson and Arah, 2020) or by delimiting the scope of the claim to focus on the outcome of an intervention to a hypothetical sample. In this case, the latter is more tractable because it is difficult to theorize how integrating occupations would change the mapping between occupations and health outcomes. 


\section{Methods: Disentangle causation from selection to estimate counterfactual health disparities}

The previous section introduced an analytical framework for studying segregation. In that framework, each person has a potential outcome in every occupation and thus an expected potential outcome if occupations were assigned equitably. However, it is only possible to observe people in the occupations they actually hold. To learn about the potential outcomes, we need to describe something in the factual world which implies (under assumptions) an answer about the counterfactual world. This section details that process.

Data come from the 2005-2020 Current Population Survey (hereafter CPS) on the civilian U.S. population ages 25-60, harmonized by the Integrated Public Use Microdata Series (IPUMS, Flood et al. 2020). The outcome variable is whether each individual reports "a health problem or a disability which prevents him/her from working or which limits the kind or amount of work." I refer to this as a report of a work-limiting disability. 4 Occupation (the treatment) is measured in 3-digit codes harmonized by IPUMS (Flood et al., 2020) to a standard based on the 2010 Census coding system, which contains 451 unique non-military occupations (e.g. chefs and cooks, elementary and middle school teachers, cashiers, roofers). Racial category is self-reported (non-Hispanic Black, non-Hispanic white, Hispanic, other). The data also contain covariates including sex (men, women), education (less than high school, high school, some college, college), foreign born, age, year, and a lagged measure of the standard five-point scale of self-rated health. ${ }^{5}$

\footnotetext{
${ }^{4}$ This measurement of work-limiting disability is widely used in studies using the CPS (e.g. Brucker et al. 2015; Jajtner et al. 2020) as well as studies relying on similar questions in the Health and Retirement Study (e.g. Burkhauser et al. 1999), the Panel Study of Income Dynamics (e.g. Meyer and Mok 2019), and the National Longitudinal Survey of Youth (e.g. Pais 2014). Appendix E discusses limitations of this operationalization of work-limiting disability: it only captures respondent perceptions of their own health, and the CPS survey instrument changed slightly over time.

${ }^{5}$ Supplementary analyses contain a more thorough adjustment set at the cost of narrowing the scope of years involved. Beginning in 2009, the CPS added a series of six questions in which respondents reported whether they had any difficulty with hearing, vision, remembering, walking or climbing stairs, performing basic activities outside the home alone, or taking care of their personal needs. Analyses on the subset of people who answer no to all of those questions yield substantively similar results (Appendix Fig 16 Panel C).
} 
All predictor variables (racial category, occupation, and covariates) are measured at year t. The outcome is measured 12 months later, taking advantage of the linked panel structure of the CPS (Appendix Fig. 13, see Rivera Drew et al. 2014). The main analyses restrict the sample to those who report at year $t$ that they have never quit a job for health reasons, are currently employed, and do not have a work-limiting disability (Fig 4). This restriction on the lagged outcome and employment reduces the risk that differences in the outcome observed one year later are consequences of latent health conditions. The analytical sample contains 247,847 persons. For each person in the sample, the empirical task can be broken into two components: making an estimate for each person and then aggregating over people.

We first want to know the outcome that person would realize if assigned an occupation from the equitable rule. Consider a subpopulation in which every person has exactly the covariate set of person $i$. Within that subpopulation, there is variation in occupations and in health outcomes. To make an estimate for person $i$, we could take a weighted average of the health outcomes of all the people with covariates that match person $i$, where the weights correspond to the counterfactual occupational distribution of interest. Doing so answers a descriptive question: what health outcome is observed for people who look like person $i$, but who hold different occupations? We might be willing to make the additional assumption that the outcome is a good estimate for what would happen to person $i$ if counterfactually assigned to an occupation by the equitable rule. That assumption is depicted in the Directed Acyclic Graph in Fig. 5A and may be credible because this population is identical along lagged health, demographic, and human capital variables so that the most obvious sources of selection bias are blocked. For instance, the adjustment set accounts for unequal selection into occupations on the basis of lagged work-limiting disability or on the basis of education. One of the main threats to identification is that lagged health (an important confounder) is a latent construct for which lagged reports of health are a coarse measurement; Appendix $\mathrm{C}$ discusses two forms of this threat. The threat is mitigated in part by the fact that lagged health is reported with several constructs, one of which involves the exact same question as 


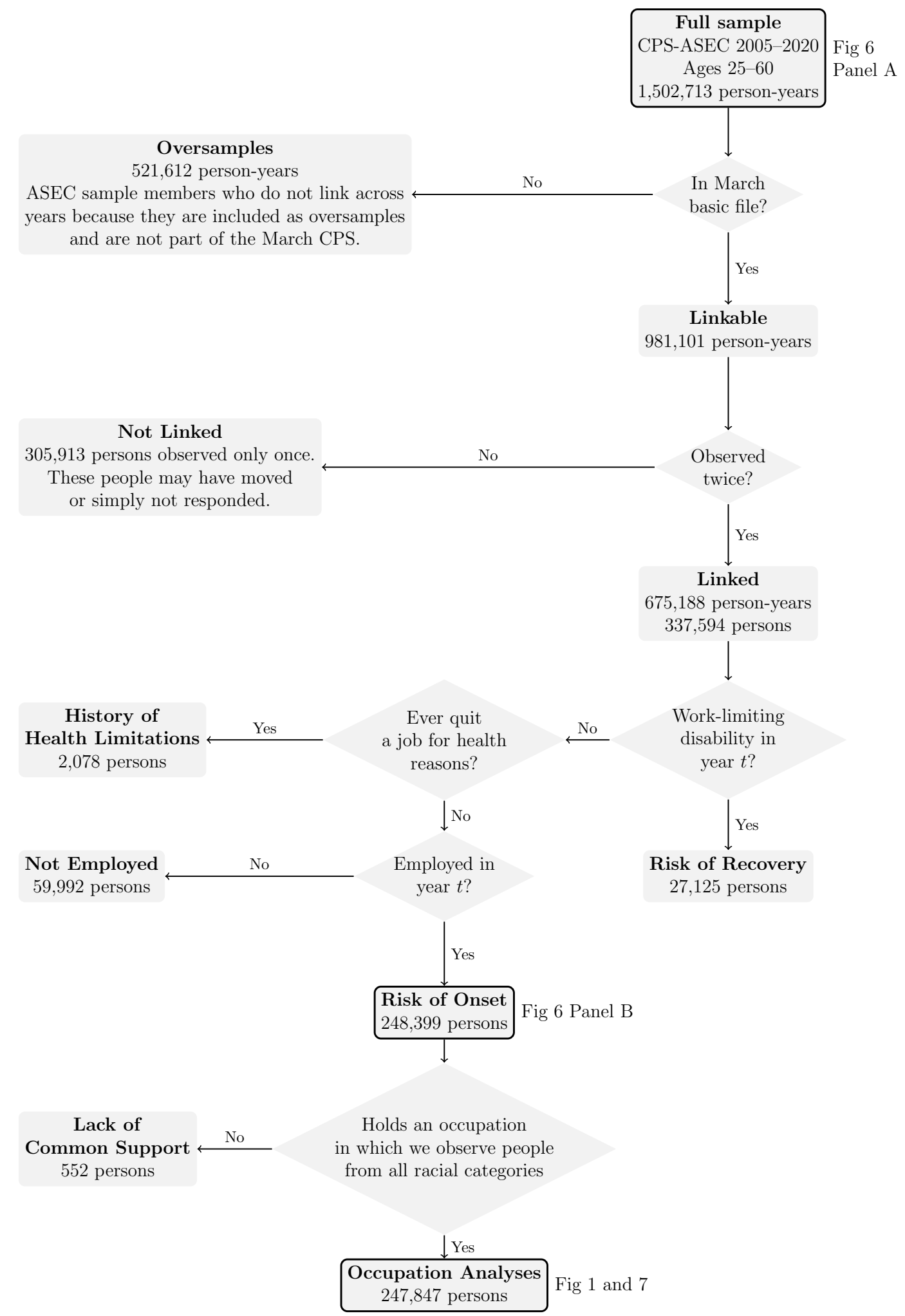

Fig. 4. Sample restrictions. The three rectangles with black outlines correspond to samples analyzed in the main text. 
A) Make causal assumptions in a Directed Acyclic Graph (Pearl, 2009)

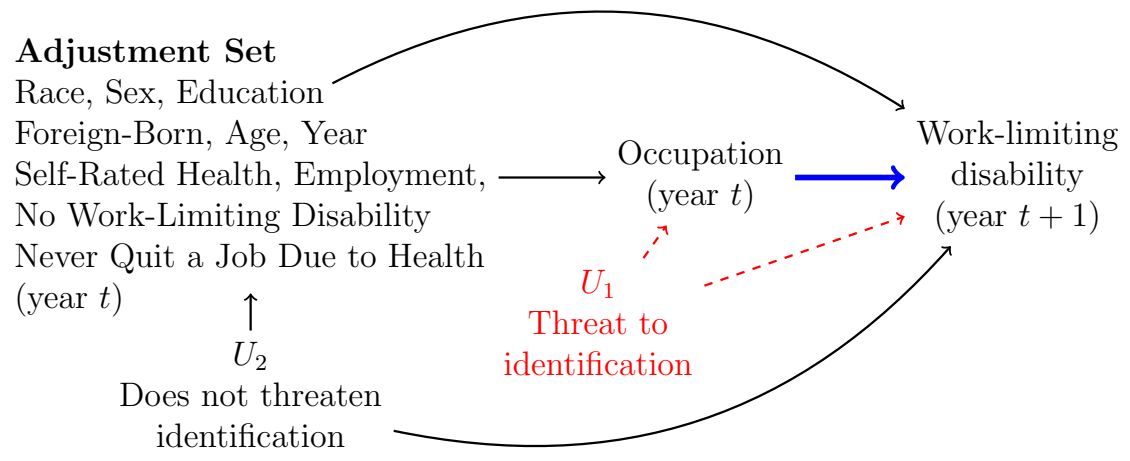

B) Fit a prediction function for the outcome

$$
f(x, \vec{\ell}, d)=\mathrm{P}(Y=1 \mid \text { Category }=x, \text { Covariates }=\vec{\ell}, \text { Occupation }=d)
$$

Probability of disability onset given racial category, covariates, and occupation

C) Predict counterfactuals for each unit $i$ using the estimated function $\hat{f}()$.

Counterfactuals are defined over occupation only; keep other predictors as observed.

Predict for each occupation $d$.

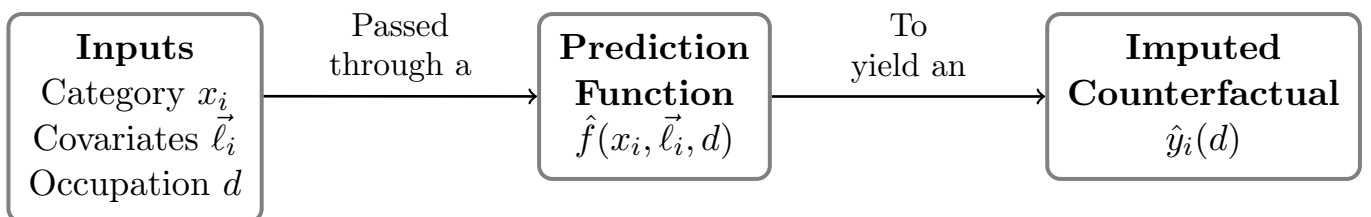

D) For each person $i$, average over the counterfactual occupation assignments

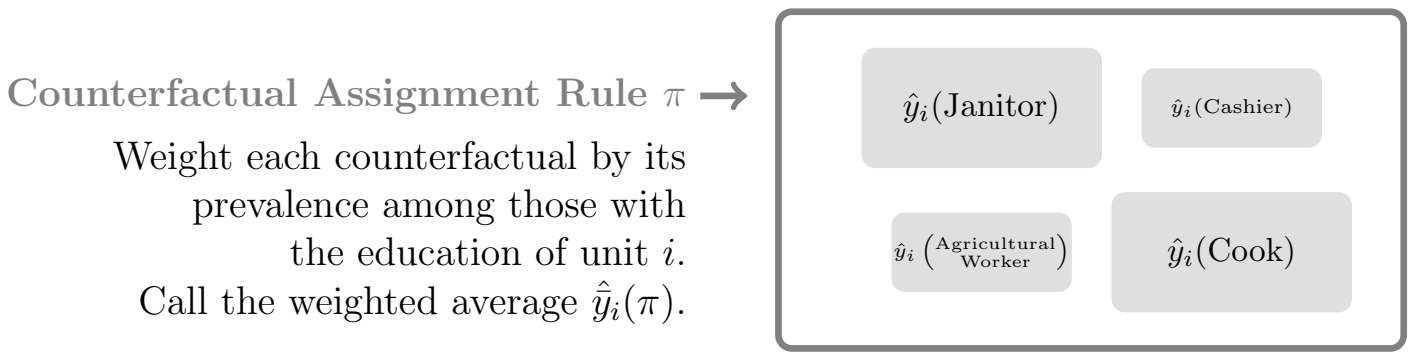

Example: Four occupations sized by prevalence among those who did not complete high school.

E) Report a sample-weighted average of $\hat{\bar{y}}_{i}(\pi)$ over people $i$ in each racial category.

Fig. 5. Procedure to estimate counterfactual disparities. Under causal assumptions (A), a predictive algorithm (B) can impute the counterfactual probability of work-limiting disability onset that each person $i$ would realize if exposed to any occupation $d(\mathrm{C})$. Weighting those counterfactuals by a new rule for assigning occupations (D) and averaging over subpopulations (E) yields counterfactual disparity estimates. 
the one by which the outcome is measured.

The argument above has conceptually focused on a subpopulation that is identical to person $i$ along all of the covariates. In practice, that subpopulation may be extremely small in the data. Instead of actually creating subpopulations, I estimate a prediction function for the mean outcome that we would observe if we found people identical to person $i$. Specifically, I estimate a Generalized Additive Model (GAM, Wood 2017), which is analogous to a generalized linear model but allows non-linear terms for continuous predictors and supports hierarchical methods for more efficient estimation of the occupation-specific intercepts. I estimate four separate models on subpopulations defined by the four racial categories, thereby allowing the function mapping predictors to outcomes to differ across race. Appendix D presents modeling details. Given the predictive model (Fig. 5B), I make predictions for each person at their observed covariates but at each possible occupation (Fig. 5C). Then, I take a weighted average for each person $i$ over occupations (Fig. 5D). The strategy of using a predictive algorithm rather than strata of the population is advantageous because it improves the precision of estimates by sharing information from people with similar covariate values.

Once we have an estimate for each person, we can proceed to the second component of estimation: averaging over people (Fig. 5E). At this point, we are not comparing (for example) Black and white people who are identical along all covariates, as one would in a regression model. Instead, we are comparing Black and white people who differ along all the covariates as those populations differ in the real world. The covariates are used to make an estimate for each person, but the average of those person-specific estimates across populations does not hold the covariates constant. This makes the goal of the empirical analysis different from the goal of a single regression model. The final estimates do not hold all covariates constant; they emulate a hypothetical intervention to occupation alone. 
A) In the full population, work-limiting disabilities exhibit large disparities by race and ethnicity

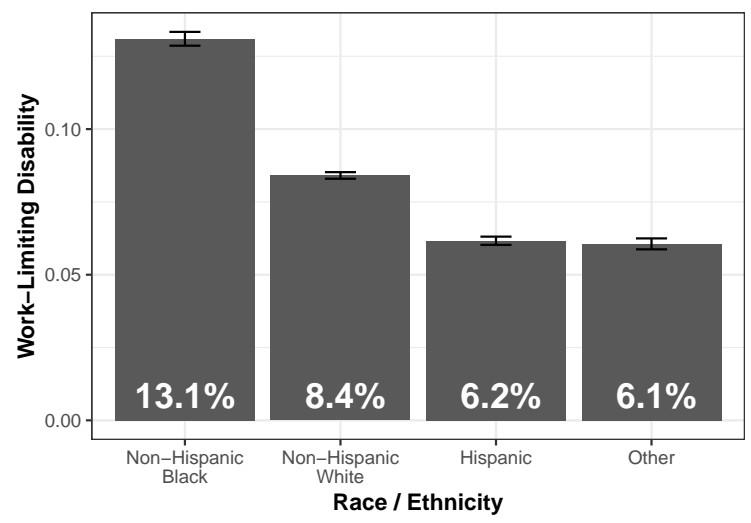

B) Among those employed with no work-limiting disability this year, there exist severe disparities in onset of disability next year

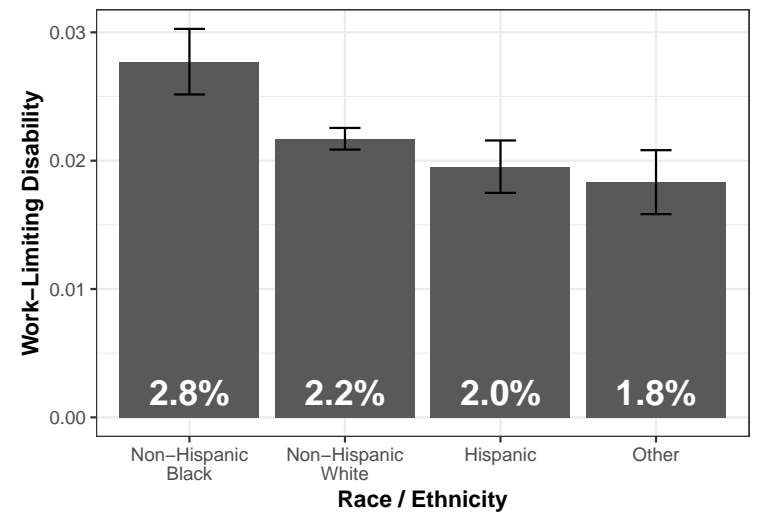

Fig. 6. Disparities in work-limiting disability across population subgroups. Each panel pools data from the 2005-2020 March Supplement of the Current Population Survey. Error bars depict $95 \%$ confidence intervals based on variance estimates calculated using replicate weights (Appendix F).

\section{$5 \quad$ Results}

Among the U.S. civilian population ages 25-60 in 2005-2020, work-limiting disabilities are common and disparate across categories of race/ethnicity: a work-limiting disability is reported for $13.1 \%$ of those who identify as non-Hispanic Black but only $8.4 \%$ of non-Hispanic whites, $6.2 \%$ of Hispanics, and $6.1 \%$ of those who identify with another racial category (Fig. 6 Panel A). Focusing on the subpopulation who are employed and do not report a disability in year $t$, there are also disparities in the onset of disability by year $t+1$, which is reported by $2.8 \%$ of non-Hispanic Black workers compared with only $2.2 \%$ of non-Hispanic white workers, $2.0 \%$ of Hispanic workers, and $1.8 \%$ of those who identify with another racial category (Fig. 6 Panel B). Although the prevalence of disability onset (Panel B) is lower than the overall prevalence of disability (Panel A), focusing on onset is useful because it directs attention to the process by which those overall disparities arise.

To what degree are the disparities in Fig 6 Panel B a consequence of occupational seg- 
regation? Fig 7 summarizes the onset of work-limiting disability in the factual population and under the counterfactual assignment rule. Equalizing occupational assignments within education categories would reduce the onset of work-limiting disability among non-Hispanic Black individuals from $2.8 \%$ to $2.6 \%$ and increase it slightly among non-Hispanic white individuals (who are now exposed to riskier occupations) (Panel A). The counterfactual Black-white disparity is $36 \%$ smaller than the factual disparity: a reduction from a disparity of 0.6 percentage points to 0.4 percentage points (Panel B). A reduction of 0.2 percentage points corresponds to a large number of people: it implies that roughly 39,200 Black workers who experienced onset of a work-limiting disability in a given year would not have done so if occupations were allocated equitably. ${ }^{6}$ Unequal allocation to occupations is therefore responsible for some of the Black-white disparity in the onset of work-limiting disability. But the amount accounted for by occupation is far from all of the racial disparity. Structural racism shapes health in many forms, and other contributors to the racial disparity (e.g. neighborhood environments) expose people to differential hazards outside of work. Occupational segregation is a substantial part of the story, but it is not the whole story.

The two other counterfactual disparities (Hispanic vs. non-Hispanic white and other vs. non-Hispanic white) follow a different pattern. Each of these factual disparities runs in the opposite direction of the Black-white disparity: these groups have lower onset of worklimiting disability compared to non-Hispanic whites. Yet they also work in more dangerous occupations. Equalizing occupational assignments would increase the Hispanic-white gap by $70 \%$ and the other-white gap by $34 \%$. The ability to make those claims is a key benefit of a causal perspective: even though these groups have descriptively better health than non-Hispanic whites, we would expect their health to be even better in a counterfactual world where occupations were allocated equitably. Hispanic individuals in the U.S., for example, possess other covariates that are associated with good health, such as a younger

\footnotetext{
${ }^{6}$ In the fourth quarter of 2019 there were 19.6 million employed Black Americans (Edwards and Smith, 2020). The above estimate (based on 2005-2020) implies that $0.2 \%$ of these individuals experience disability onset and would not have experienced that onset if occupations were allocated equitably. That corresponds to $0.2 \% \times 19.6$ million $=39,200$ people.
} 
A) Equalizing occupational assignments for a sample would reduce the onset of worklimiting disability for all groups except non-Hispanic whites

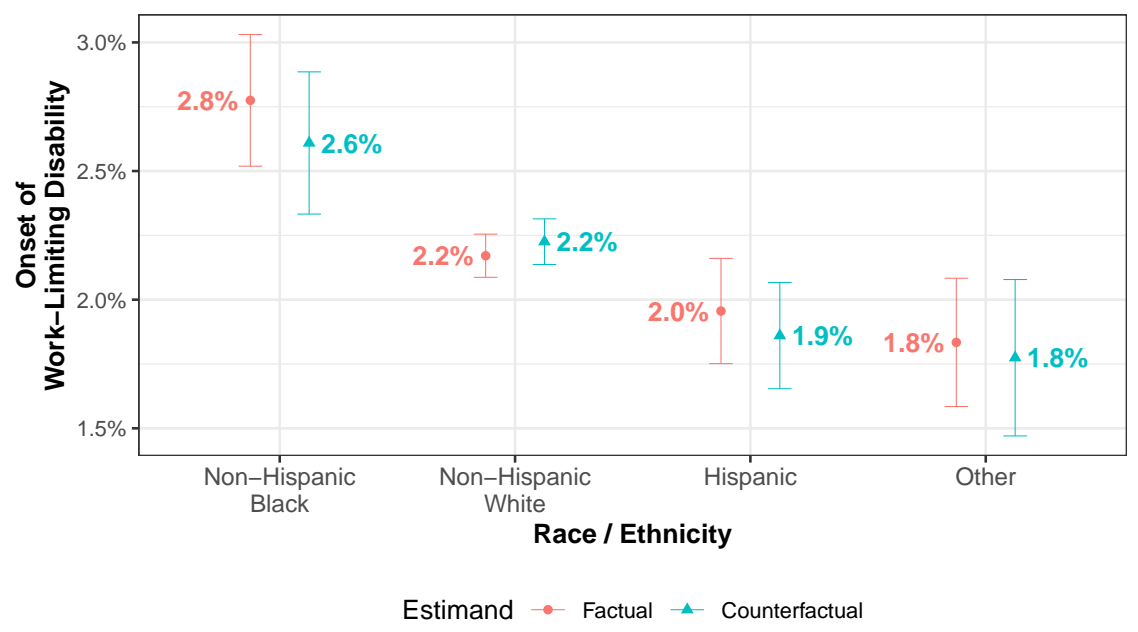

B) Equalizing occupational assignments for a sample would reduce the Black-white disparity by $36 \%$. It would increase the health advantage of the other categories compared to non-Hispanic whites.

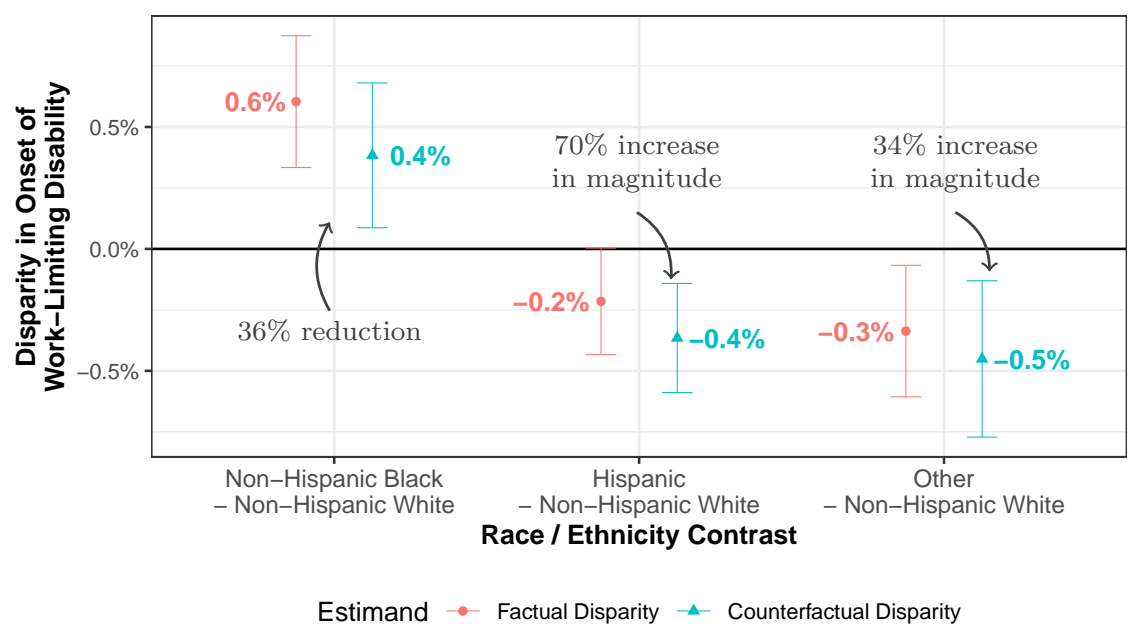

Fig. 7. Factual and counterfactual probabilities of work-limiting disability onset. The counterfactual quantities refer to the subgroup average of the individual average potential outcome realized if occupations were assigned proportional to their prevalence within one's educational category. In Panel B, the $36 \%$ reduction and $70 \%$ increase are statistically significantly different from zero at the 0.05 level. 
age distribution and a higher proportion immigrants. ${ }^{7}$ They do not fully realize the better health we might expect given those covariates in part because they are employed in more hazardous occupations. Just because a category has good health does not imply that the labor market treats that category well.

\section{Discussion}

Racial health disparities are partially rooted in economic structures. That was true when the American economy rested on the backs of slaves. It was true in the industrial tragedy of the Hawk's Nest Tunnel, when a company profited by drilling through a mountain at breakneck speed by neglecting even the most basic protections for the workers involved. And it is true today, as people of color continue to work disproportionately in the occupations with the greatest hazards.

This paper presented three claims. First, work-limiting disabilities are highly disparate by race. Among Americans ages 25-60, only 8.4\% of those identifying as non-Hispanic white report a health condition that limits the kind or amount of work they can do. The same statistic among those identifying as non-Hispanic Black is 13.1\% (Fig 6). Drawing on panel data linked over periods of 12 months, this paper focused attention on inflows into those statistics: the onset of work-limiting disability in year $t+1$ among those who were employed without a work-limiting disability in year $t$. The onset of work-limiting disability is highest among those employed in occupations with high proportions non-Hispanic Black or Hispanic (Fig 1). Finally, Fig 7 presents this paper's central claim: if we took a sample from the population and reallocated occupations equitably (as a function of education alone), the Black-white gap in the onset of work-limiting disability would close by $36 \%$ for that sample. The health advantages of the Hispanic population and of other categories relative to nonHispanic whites would become more pronounced. In short, the unequal assignment of people

\footnotetext{
${ }^{7}$ In an alternative specification restricted to the native born population, the onset of work-limiting disabilities is factually slightly higher for Hispanics compared with non-Hispanic whites (Appendix Fig 16 Panel D).
} 
to occupations plays an important role in the production of racial disparities in work-limiting disabilities.

While important, occupational segregation is far from the whole story. In fact, it accounts for less than half of the total disparity in the onset of work-limiting disabilities. Racism in America involves many structural components, ranging from occupational segregation to residential environments to personal experiences of racism. That is why quantifying the magnitude of one specific contributor is so important: we now know that occupational segregation alone is responsible for roughly one-third of the disparity in onset of work-limiting disabilities. Eliminating occupational segregation would likely move us in the direction of health equity, but even a perfect policy to desegregate occupations would be incomplete in the absence of interventions to other causes.

These results are informative despite the unavoidable limitations of drawing causal claims from observational data. While the adjustment set is surely insufficient to block all forms of selection, incorporating lagged indicators of health and employment into the estimation procedure moves us toward a causal claim. It is infeasible to actually randomize occupations, and natural experiments with exogenous shocks to occupation would speak only to one specific context. For the goal of producing a claim representative of the population, observational data is the only option. In this setting, adjustment for confounding variables yields a useful claim even if it is imperfect. Results speak most directly to a local sampling-based interpretation: what would happen if we reallocated occupations for a sample. To make a global claim about what would happen under an economy-wide restructuring to eliminate occupational segregation, one would have to make many additional assumptions about how actors interact with each other. Absent those assumptions, the local claim provides an important point of empirical evidence from which those who desire to do so can theorize about what might happen under a more global intervention. 


\subsection{Implications for inequality and population health}

Scholars of population health have long emphasized race as a fundamental causes of disease (Link and Phelan, 1995; Phelan and Link, 2015; Williams et al., 2019). Racism is too complex to be appropriately operationalized by the coefficient on race with a set of predictors held equal. Further, analyses that seek to jointly assess the contributions of many components of racism (e.g. residential segregation and occupations and discriminatory experiences) are bound to yield incomplete understanding: each of these inputs is sufficiently complicated to require an analysis all its own. Drawing on causal approaches (Jackson and VanderWeele, 2018; Lundberg, 2021; VanderWeele and Robinson, 2014), this paper illustrates what one can learn by focusing on one input: occupational segregation. For scientific understanding of the Black-white disparity in work-limiting disabilities, it is helpful to know that occupational segregation is responsible for about one-third of that disparity. Future work could undertake similar analyses for other structural causes.

By emphasizing occupational segregation, this paper directs attention to an overlooked aspect of health: the allocation of people to occupations. The safety of each occupation has long been the focus of government policy. For example, the mission of the Occupational Safety and Health Administration is "to assure so far as possible every working man and woman in the Nation safe and healthful working conditions," (OSH Act of 1970, Section 2). The agency does that by enforcing rules about the safety of occupations. But there is an additional set of policies one could also consider which might focus on providing all people with equal access to occupations, such as training programs and enforcement of laws against hiring discrimination. Those types of policies would not eliminate racial health disparities, but they might address some of the one-third of the Black-white disparity attributed to occupational segregation in this paper. In short, future research and policy should not only explore how to reduce risks (make occupations safer) but also how to ensure that risks are distributed equitably across population subgroups (e.g. reduce barriers to occupational entry). 


\subsection{Implications for quantitative methodology}

A recurring question in sociological methodology is the linkage between micro- and macrolevel processes. One can study macro-level processes by remaining entirely at the macro level: how population-level outcomes vary across social contexts (the unit of analysis) with different structural conditions. But sometimes we do not observe any macro-level context with the structural conditions of interest (e.g. racially integrated occupations). Comparisons across contexts are also limited when variables of interest (e.g. race) have definitions that may vary across those contexts. By focusing on the outcomes of individuals exposed to different structural conditions (e.g. assigned to different occupations), one gains opportunities to explore new theoretical questions, like the outcome of an equitable rule for assigning occupations which the researcher defines. One also gains new empirical opportunities: causal claims require us to find units who are similar but with different treatment conditions, and that task is easier when the unit is an individual rather than a population because there may be hundreds of thousands of individuals but only a handful of populations. There is much to be gained by defining the consequences of a macro-level phenomenon (e.g. segregation) in terms of individual-level outcomes. The only cost of doing so comes in interpretation: in the absence of strong theoretical knowledge about interference, we must scale back the scope of claims to focus on what would happen for a sample (rather than the whole population) exposed to alternative structural conditions. Macro-level analyses have an important place in scholarship, but for many questions new insights may be possible through complementary approaches that consider the explicitly causal micro-level outcomes that individuals would experience if exposed to different macro-level conditions.

Because a pivot to micro-level data potentially unlocks sample sizes that are orders of magnitude larger (individuals instead of societies), this approach creates new opportunities for estimation via predictive algorithms as part of the growing field of computational social

science (Edelmann et al., 2020; Lazer et al., 2020). In this paper, the predictive algorithm was a Generalized Additive Model (Wood, 2017) applied separately in subpopulations defined by 
race, and used to predict unobserved potential outcomes under transparent causal assumptions. Formalizing macro-level questions in terms of a population average of unit-specific potential outcomes (as in the general framework of Lundberg et al. 2021) thus connects predictive algorithms to a macro-level sociological question.

\subsection{Conclusion}

The path to health equity requires that we understand the root causes of racial disparities. One of those root causes is occupational segregation. The Hawk's Nest Tunnel was one egregious example, but the systematic placement of people of color into harm's way by virtue of their occupational assignments is ubiquitous in the American economy. Occupational segregation accounts for roughly one-third of the racial disparity in the onset of work-limiting disabilities. Steps to eliminate segregation would move us part of the way toward health equity. Additional scholarship targeting other counterfactual health disparities under modified structural conditions could help scholarship to contribute to policy changes that might advance a more equitable future. 


\section{References}

Ahonen, E. Q., K. Fujishiro, T. Cunningham, and M. Flynn 2018. Work as an inclusive part of population health inequities research and prevention. American Journal of Public Health, 108(3):306-311.

Banzhaf, S., L. Ma, and C. Timmins 2019. Environmental justice: The economics of race, place, and pollution. Journal of Economic Perspectives, 33(1):185-208.

Bearman, P. S., J. Moody, and K. Stovel 2004. Chains of affection: The structure of adolescent romantic and sexual networks. American Journal of Sociology, 110(1):44-91.

Bertrand, M. and S. Mullainathan 2004. Are emily and greg more employable than lakisha and jamal? a field experiment on labor market discrimination. American Economic Review, 94(4):991-1013.

Blau, P. M. and O. D. Duncan 1967. The American Occupational Structure. New York: Wiley.

Brucker, D. L., S. Mitra, N. Chaitoo, and J. Mauro 2015. More likely to be poor whatever the measure: Working-age persons with disabilities in the United States. Social Science Quarterly, 96(1):273-296.

Buchanan, S., P. Vossenas, N. Krause, J. Moriarty, E. Frumin, J. A. M. Shimek, F. Mirer, P. Orris, and L. Punnett 2010. Occupational injury disparities in the U.S. hotel industry. American Journal of Industrial Medicine, 53(2):116-125.

Bureau of Labor Statistics 2006. Design and Methodology: Current Population Survey Technical Paper 66. U.S. Department of Labor.

Bureau of Labor Statistics 2020. Employer-reported workplace injuries and illnesses-2019. U.S. Department of Labor News Release, 20-2030.

Burgard, S. A. and K. Y. Lin 2013. Bad jobs, bad health? how work and working conditions contribute to health disparities. American Behavioral Scientist, 57(8):1105-1127.

Burkhauser, R. V., J. Butler, Y.-W. Kim, and R. R. Weathers 1999. The importance of accommodation on the timing of disability insurance applications: Results from the survey of disability and work and the Health and Retirement Study. Journal of Human Resources, Pp. 589-611.

Burkhauser, R. V., M. C. Daly, A. J. Houtenville, and N. Nargis 2002. Self-reported work-limitation data: What they can and cannot tell us. Demography, 39(3):541-555.

Cherniack, M. 1986. The Hawk's Nest Incident: America's Worst Industrial Disaster. New Haven, CT: Yale University Press.

Coleman, J. S. 1990. Foundations of Social Theory. Harvard University Press.

Czajka, J. L., R. Rosso, et al. 2015. Redesign of the income questions in the Current Population Survey Annual Social and Economic Supplement: Further analysis of the 2014 split-sample test. Mathematica Policy Research.

Du Bois, W. E. 1899. The Philadelphia Negro: A Social Study. Philadelphia: University of Pennsylvania Press.

Edelmann, A., T. Wolff, D. Montagne, and C. A. Bail 2020. Computational social science and sociology. Annual Review of Sociology, 46:61-81.

Edwards, R. and S. M. Smith 2020. Job market remains tight in 2019, as the unemployment rate falls to its lowest level since 1969. Bureau of Labor Statistics: Monthly Labor Review, April.

Fay, R. E. and G. F. Train 1995. Aspects of survey and model-based postcensal estimation of income and poverty characteristics for states and counties. In Proceedings of the Section on Government Statistics, American Statistical Association, Alexandria, VA, Pp. 154-159.

Ferguson, J.-P. and R. Koning 2018. Firm turnover and the return of racial establishment segregation. American Sociological Review, 83(3):445-474.

Fleischer, N. L., H. M. Tiesman, J. Sumitani, T. Mize, K. K. Amarnath, A. R. Bayakly, and M. W. Murphy 2013. Public health impact of heat-related illness among migrant farmworkers. American Journal of Preventive Medicine, 44(3):199-206. 
Flood, S., M. King, R. Rodgers, S. Ruggles, and J. R. Warren 2020. Integrated Public Use Microdata Series, Current Population Survey: Version 8.0 [dataset]. Minneapolis, MN: IPUMS.

Flood, S. M. and J. D. Pacas 2017. Using the Annual Social and Economic Supplement as part of a Current Population Survey Panel. Journal of Economic and Social Measurement, 42(3-4):225-248.

Fujishiro, K., A. Hajat, P. A. Landsbergis, J. D. Meyer, P. J. Schreiner, and J. D. Kaufman 2017. Explaining racial/ethnic differences in all-cause mortality in the multi-ethnic study of atherosclerosis (mesa): Substantive complexity and hazardous working conditions as mediating factors. SSM-Population Health, 3:497-505.

Geronimus, A. T. 1992. The weathering hypothesis and the health of african-american women and infants: Evidence and speculations. Ethnicity \& Disease, 2(3):207-221.

Geronimus, A. T., M. Hicken, D. Keene, and J. Bound 2006. "Weathering" and age patterns of allostatic load scores among blacks and whites in the United States. American Journal of Public Health, 96(5):826-833.

Greenland, S. 2003. Quantifying biases in causal models: classical confounding vs collider-stratification bias. Epidemiology, 14(3):300-306.

Greenland, S., J. Pearl, and J. M. Robins 1999. Causal diagrams for epidemiologic research. Epidemiology, 10(1):37-48.

Greiner, D. J. and D. B. Rubin 2011. Causal effects of perceived immutable characteristics. Review of Economics and Statistics, 93(3):775-785.

Hale, T. W. 2001. The lack of a disability measure in today's current population survey. Monthly Labor Review, 124:38.

Harknett, K., D. Schneider, and R. Wolfe 2020. Losing sleep over work scheduling? The relationship between work schedules and sleep quality for service sector workers. SSM-Population Health, 12:100681.

Hedström, P. and P. S. Bearman 2009. The Oxford Handbook of Analytical Sociology. Oxford University Press.

Heymann, J. 2003. Global Inequalities at Work: Work's Impact on the Health of Individuals, Families, and Societies. Oxford University Press.

Holland, P. W. 1986. Statistics and causal inference. Journal of the American Statistical Association, 81(396):945-960.

Hout, M. 2012. Social and economic returns to college education in the United States. Annual Review of Sociology, 38:379-400.

Imbens, G. W. and D. B. Rubin 2015. Causal Inference in Statistics, Social, and Biomedical Sciences. Cambridge University Press.

Jackson, J. W. 2021. Meaningful causal decompositions in health equity research: definition, identification, and estimation through a weighting framework. Epidemiology, 32(2):282-290.

Jackson, J. W. and O. A. Arah 2020. Invited commentary: making causal inference more social and (social) epidemiology more causal. American Journal of Epidemiology, 189(3):179-182.

Jackson, J. W. and T. J. VanderWeele 2018. Decomposition analysis to identify intervention targets for reducing disparities. Epidemiology, 29(6):825.

Jajtner, K. M., S. Mitra, C. Fountain, A. Nichols, et al. 2020. Rising income inequality through a disability lens: Trends in the United States 1981-2018. Social Indicators Research, Pp. 1-34.

Juon, H.-S., A. Hong, M. Pimpinelli, M. Rojulpote, R. McIntire, and J. A. Barta 2021. Racial disparities in occupational risks and lung cancer incidence: Analysis of the national lung screening trial. Preventive Medicine, 143:106355.

Kang, J. D. and J. L. Schafer 2007. Demystifying double robustness: A comparison of alternative strategies for estimating a population mean from incomplete data. Statistical Science, 22(4):523-539.

Kohler-Hausmann, I. 2018. Eddie Murphy and the dangers of counterfactual causal thinking about detecting racial discrimination. Northwestern University Law Review, 113:1163. 
Krieger, N. 2001. A glossary for social epidemiology. Journal of Epidemiology $\&$ Community Health, 55(10):693-700.

Lazer, D. M., A. Pentland, D. J. Watts, S. Aral, S. Athey, N. Contractor, D. Freelon, S. Gonzalez-Bailon, G. King, H. Margetts, et al. 2020. Computational social science: Obstacles and opportunities. Science, 369(6507):1060-1062.

Lewis, T. T., C. D. Cogburn, and D. R. Williams 2015. Self-reported experiences of discrimination and health: scientific advances, ongoing controversies, and emerging issues. Annual Review of Clinical Psychology, 11:407-440.

Link, B. G. and J. Phelan 1995. Social conditions as fundamental causes of disease. Journal of Health and Social Behavior, Pp. 80-94.

Lipscomb, H. J., D. Loomis, M. A. McDonald, R. A. Argue, and S. Wing 2006. A conceptual model of work and health disparities in the United States. International Journal of Health Services, 36(1):25-50.

Lowry, S. J., H. Blecker, J. Camp, B. De Castro, S. Hecker, S. Arbabi, N. Traven, and N. S. Seixas 2010. Possibilities and challenges in occupational injury surveillance of day laborers. American Journal of Industrial Medicine, 53(2):126-134.

Lundberg, I. 2021. The gap-closing estimand: A causal approach to study interventions that close disparities across social categories. SocArXiv manuscript: https://doi.org/10.31235/osf.io/gx4y3.

Lundberg, I., R. Johnson, and B. M. Stewart 2021. What is your estimand? Defining the target quantity connects statistical evidence to theory. SocArXiv manuscript: https://doi.org/10.31235/osf.io/ ba67n.

Maestas, N., K. J. Mullen, and S. Rennane 2019. Unmet need for workplace accommodation. Journal of Policy Analysis and Management, 38(4):1004-1027.

Marmot, M. and R. Wilkinson 2005. Social Determinants of Health. Oxford University Press.

McEwen, B. S. 1998. Protective and damaging effects of stress mediators. New England Journal of Medicine, 338(3):171-179.

Merkin, S. S., R. Basurto-Dávila, A. Karlamangla, C. E. Bird, N. Lurie, J. Escarce, and T. Seeman 2009. Neighborhoods and cumulative biological risk profiles by race/ethnicity in a national sample of us adults: Nhanes iii. Annals of Epidemiology, 19(3):194-201.

Meyer, B. D. and W. K. Mok 2019. Disability, earnings, income and consumption. Journal of Public Economics, 171:51-69.

Moen, P., E. L. Kelly, E. Tranby, and Q. Huang 2011. Changing work, changing health: can real work-time flexibility promote health behaviors and well-being? Journal of Health and Social Behavior, 52(4):404-429.

Ólafsdóttir, S. and J. Beckfield 2020. Health inequalities: What do we know and what do we need to know? Social Science \& Medicine, 267:113575-113575.

Omi, M. and H. Winant 2014. Racial Formation in the United States. Routledge.

Pais, J. 2014. Cumulative structural disadvantage and racial health disparities: The pathways of childhood socioeconomic influence. Demography, 51(5):1729-1753.

Pearl, J. 2009. Causality. Cambridge University Press.

Phelan, J. C. and B. G. Link 2015. Is racism a fundamental cause of inequalities in health? Annual Review of Sociology, 41:311-330.

Prins, S. J., S. McKetta, J. Platt, C. Muntaner, K. M. Keyes, and L. M. Bates 2019. Mental illness, drinking, and the social division and structure of labor in the United States: 2003-2015. American Journal of Industrial Medicine, 62(2):131-144.

Rivera Drew, J. A., S. Flood, and J. R. Warren 2014. Making full use of the longitudinal design of the Current Population Survey: Methods for linking records across 16 months. Journal of Economic and Social Measurement, 39(3):121-144.

Schulte, P. A., R. Pana-Cryan, T. Schnorr, A. L. Schill, R. Guerin, S. Felknor, and G. R. Wagner 2017. An 
approach to assess the burden of work-related injury, disease, and distress. American Journal of Public Health, 107(7):1051-1057.

Seabury, S. A., S. Terp, and L. I. Boden 2017. Racial and ethnic differences in the frequency of workplace injuries and prevalence of work-related disability. Health Affairs, 36(2):266-273.

Sen, M. and O. Wasow 2016. Race as a bundle of sticks: Designs that estimate effects of seemingly immutable characteristics. Annual Review of Political Science, 19.

Stainback, K. and D. Tomaskovic-Devey 2012. Documenting Desegregation: Racial and Gender Segregation in Private Sector Employment Since the Civil Rights Act. Russell Sage Foundation.

Stanbury, M. and K. D. Rosenman 2014. Occupational health disparities: A state public health-based approach. American Journal of Industrial Medicine, 57(5):596-604.

Tomaskovic-Devey, D., C. Zimmer, K. Stainback, C. Robinson, T. Taylor, and T. McTague 2006. Documenting desegregation: Segregation in american workplaces by race, ethnicity, and sex, 1966-2003. American Sociological Review, 71(4):565-588.

Torrats-Espinosa, G. 2021. Using machine learning to estimate the effect of racial segregation on covid-19 mortality in the united states. Proceedings of the National Academy of Sciences, 118(7).

VanderWeele, T. J. and W. R. Robinson 2014. On causal interpretation of race in regressions adjusting for confounding and mediating variables. Epidemiology (Cambridge, Mass.), 25(4):473.

Venables, W. N. and B. D. Ripley 2002. Modern Applied Statistics with S, fourth edition. New York: Springer. ISBN 0-387-95457-0.

Williams, D. R., J. A. Lawrence, and B. A. Davis 2019. Racism and health: Evidence and needed research. Annual Review of Public Health, 40:105-125.

Williams, D. R. and S. A. Mohammed 2009. Discrimination and racial disparities in health: Evidence and needed research. Journal of Behavioral Medicine, 32(1):20-47.

Wood, S. N. 2003. Thin plate regression splines. Journal of the Royal Statistical Society: Series B (Statistical Methodology), 65(1):95-114.

Wood, S. N. 2017. Generalized Additive Models: An Introduction with R. Chapman and Hall/CRC. 


\section{A Weighting of the CPS-ASEC}

The CPS-ASEC samples people with unequal probabilities. All analyses in the main text weight by the ASECWT weighting variable designed for the ASEC, with inference calculated from variances estimated with replicate weights (Appendix F). Supplemental analyses show similar results with the LINKFW1YWT weight provided by IPUMS for the subsample that links forward one year (Flood et al., 2020); because replicate weights are not available for this weight so that the appropriate construction of confidence intervals is not clear, results with that weight are provided in Fig 16 Panel E. Because the U.S. population grows over time, I allow the weight variable to place greater weight on the more recent years proportional to the relatively larger size of the population (see Fig 14).

In addition to weighting population averages, the analyses also weight the generalized additive models (Wood, 2017) used to estimate conditional outcomes given predictors. The reason to weight the GAM is because it is only an approximation to a conditional mean function that is likely to be more complex; in fact, there are likely to be omitted interactions that the additive aspect of the GAM will miss. The aim is for the GAM to provide a useful and parsimonious approximation to the more complex conditional mean function. In order to prioritize the quality of that approximation in regions of the covariate space that are more heavily weighted by the sampling weights (where the predictions will matter most), the GAM estimation is weighted.

\section{B Methods for descriptive scatterplots (Fig 1)}

Because of the large number of occupations, some occupations are sparsely populated. The descriptive occupation-level analyses in Fig 1 use a partial-pooling strategy to improve pre-

cision. I first score each occupation on two dimensions: (1) the racial/ethnic composition of those employed in the occupation and (2) the proportion of those employed in the occupation this year to report a work-limiting disability next year. I then estimate occupation-level sum- 
maries with bivariate person-level regression models for the outcome of interest (e.g. onset of work-limiting disability) as a function of occupation random intercepts, thereby partially pooling information across occupations and improving efficiency by pulling estimates (particularly for small occupations) toward the overall mean. I repeat this process for each occupational summary of interest: the proportion with onset of work-limiting disability next year, the proportion non-Hispanic Black, the proportion Hispanic, and the proportion identifying with other racial categories (non-Hispanic white omitted). The scatterplots visualize occupation-level onset of work-limiting disability plotted against occupation-level racial composition. The best-fit line is a linear regression through the estimated points (occupations), with each occupation weighted proportional to size.

\section{Threats to causal identification}

Causal identification from observational data requires assumptions (Fig 5A), and those assumptions are never perfect. This section discusses two threats to identification, both of which derive in part from the fact that lagged survey reports of health coarsely capture a latent construct. Both threats are mitigated in part by the adjustment for several measures of lagged health in the analyses: adjustment for lagged self-rated health and restriction of the sample to those who never quit a job for health reasons and who do not initially report a work-limiting disability (the lagged outcome).

Despite these adjustments, latent health remains a possible confounder (Fig 8). If it were possible to know a true continuous and multi-dimensional construct of latent health, it is likely that latent health would cause occupation in year $t$ and the health outcome in year $t+1$ through paths not fully captured by the measures of lagged health. Because health is intrinsically difficult to measure, this threat is unavoidable. Nonetheless, adjustment for lagged reports of health may substantially reduce the magnitude of the bias, because those lagged reports may capture the primary pathways through which latent health affects the 


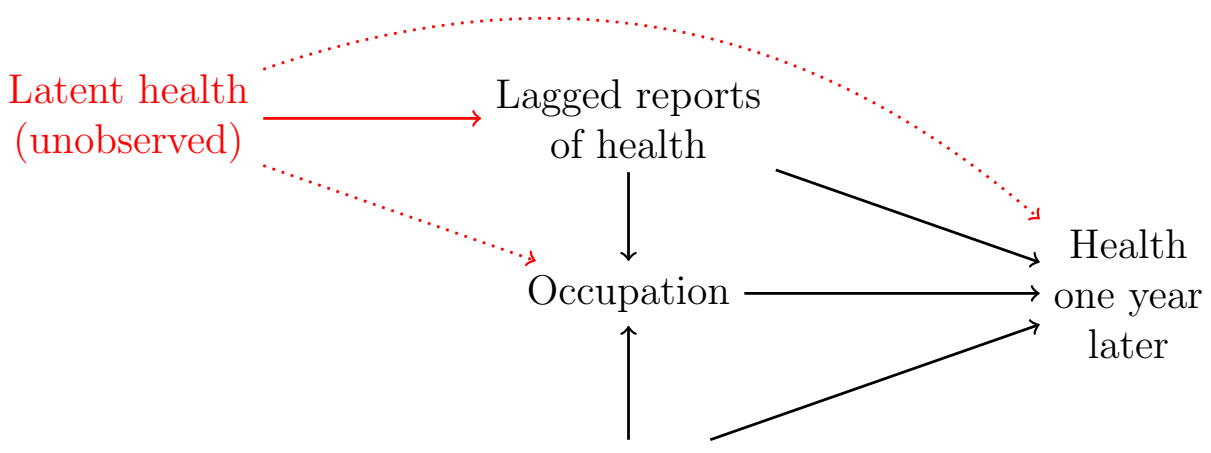

Other covariates

Fig. 8. Threat to causal identification: Coarse measurement of lagged health. The lagged health reports are likely to be coarse measures of latent health. To the degree that uncaptured components of latent health directly affect health outcomes and the occupation one holds (dotted edges), identification will be imperfect. This risk is mitigated to the degree that the lagged reports of health capture the relevant aspects of latent health that affect occupations and affect the health outcome, so that the dotted edges may be relatively unimportant.

treatment (occupation) and the outcome (health one year later).

A second threat arises from the possibility that lagged health is a pre-treatment collider variable (Fig. 9). Because the CPS panel can only be linked over two years, I observe occupation in years $t$ and $t+1$ but not in year $t-1$. It is likely that occupation in year $t-1$ causes occupation in year $t$. If occupations cause health outcomes (the main claim of this paper), then occupation in year $t-1$ affects lagged reports of health from year $t$. Lagged health is a collider variable because it is also a consequence of latent health conditions. If those latent health conditions cause health one year later through pathways other than the observed measures of health, then there is a danger of $M$-bias: holding lagged reports of health constant induces an association between lagged health and the occupation held at $t-1$, thus opening a backdoor path which creates confounding (Greenland, 2003; Greenland et al., 1999). Intuitively, suppose that in order to have no work-limiting disability at year $t$ (one of the lagged reports of health) you either have to hold a safe occupation or you have to have very good latent health. Among those with no work-limiting disability at year $t$, those in the most dangerous occupations would then have the best latent health. That 


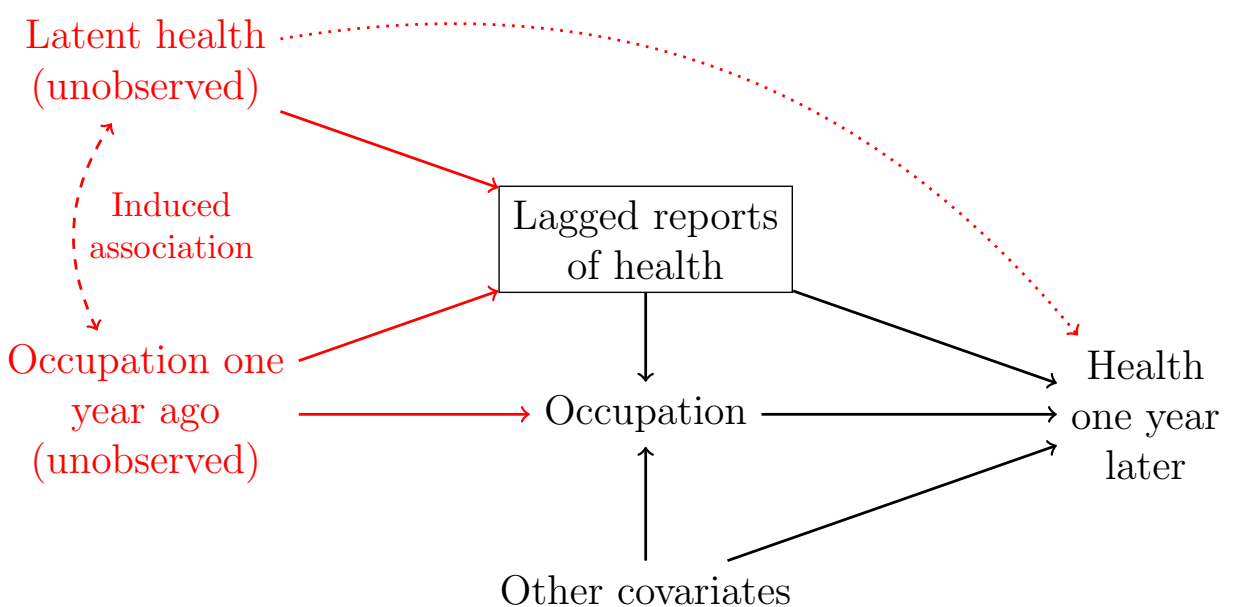

Fig. 9. Threat to causal identification: $M$-bias. Lagged health is a collider variable because it is likely to be a consequence of both latent health and the occupation one a year ago. Conditioning on lagged health induces an association between those causes. This opens a backdoor path (dashed) that would confound estimates of the causal effect of occupation on health one year later. This risk is mitigated to the degree that the lagged reports of health capture the relevant aspects of latent health that affect the health outcome, so that the dotted edge may be relatively unimportant.

induced association could cause the results to underestimate the contribution of occupational segregation to health, because the most dangerous jobs are filled with the people who are healthiest in unobserved ways.

Despite the possibility of inducing some $M$-bias, adjustment for lagged reports of health is worthwhile because it mitigates the most serious threat to identification: that lagged health affects future health and affects the occupation one holds. Further, each of these threats is substantially diminished to the degree that the lagged reports of health capture the aspect of latent health most relevant to the outcome variable. Because the lagged reports of health include a lagged dependent variable, the threats may be reasonably small. For these reasons, estimates are useful despite these limitations. 


\section{Details of how prediction functions are specified and used to estimate the main results}

This section provides details about the methods applied to the CPS-ASEC to yield the main estimates of this paper. Under the identifying assumptions in the DAG presented in Fig 5A, the observed data are informative about the unobserved data. In particular, we can predict the probability of disability $\hat{y}_{i}(d)$ that each person $i$ would experience if counterfactually exposed to occupation $d$, based on the health outcomes actually observed among those who share their race and covariate values $\vec{\ell}$ and are factually exposed to occupation $d$. The task of a statistical prediction function is simply to predict health outcomes as a function of the category (race), all covariates (e.g. education), and occupation. Because racial disparities are central to the research question, I estimate separate models on subsamples defined by racial categories. This means that the relationship between the predictors and the outcome is allowed to vary by race. The model a Generalized Additive Models (GAM, Wood 2017) specified as by Eq. D.1.

$$
\begin{aligned}
& \mathbf{E}\left(\text { Onset of Work-Limiting Disability } \mid \text { Occupation }_{i}, \text { Covariates }_{i}, \text { Race }_{i}=r\right. \text { ) } \\
& \text { Occupation-specific } \\
& \text { intercept (see D.2) } \\
& =\overbrace{\alpha_{\text {Occupation }_{i}}^{r}}+\beta_{1}^{r}\left(\text { Education }_{i}=\text { Less than High School }\right) \\
& +\beta_{2}^{r}\left(\text { Education }_{i}=\text { Some College }\right)_{+} \beta_{3}^{r}\left(\text { Education }_{i}=\text { College }\right) \\
& +\beta_{4}^{r}\left(\text { Self-Rated Health }{ }_{i}=\text { Poor }\right)+\beta_{5}^{r}\left(\text { Self-Rated Health }{ }_{i}=\text { Fair }\right) \\
& +\beta_{6}^{r}\left(\text { Self-Rated Health }_{i}=\text { Good }\right)+\beta_{7}^{r}\left(\text { Self-Rated Health }{ }_{i}=\text { Very Good }\right) \\
& +\beta_{8}^{r}\left(\text { Sex }_{i}=\text { Female }\right)+\beta_{9}^{r}\left(\text { Foreign-Born }_{i}=\text { True }\right) \\
& +\underbrace{\beta_{10}^{r}\left(\operatorname{Year}_{i} \geq 2014\right)}_{\begin{array}{c}
\text { Intercept shift when the } \\
\text { survey structure changed } \\
\text { (see Appendix E) }
\end{array}}+\underbrace{s_{\text {Year }}^{r}\left(\operatorname{Year}_{i}\right)+s_{\text {Age }}^{r}\left(\operatorname{Age}_{i}\right)}_{\begin{array}{c}
\text { Smooth functions of year and age } \\
\text { (thin-plate splines) }
\end{array}}
\end{aligned}
$$

Every term has an $r$ superscript because the model is estimated separately by race. The 
smooth terms $s()$ are thin-plate splines (Wood, 2003) to allow a smooth but possibly nonlinear functional form for continuous predictors. The indicator for ( Year $_{i} \geq 2014$ ) allows a discontinuity at 2014 because of a change in questionnaire design at that point (Appendix E). As discussed in Section 4, the model implicitly conditions on lagged reports of no worklimiting disability (a lagged dependent variable) and never quitting a job for health reasons by restricting the sample along these variables.

An important choice is how to estimate the occupation-specific intercepts $\alpha_{d}^{r}$. Estimates could be very uncertain if I estimated an unregularized coefficient on an indicator variable for each occupation, because in some occupations the number of people observed is small. A standard multilevel model would improve precision by regularizing the occupation-specific intercept $\alpha_{d}^{r}$ toward the grand mean. But the entire motivation for the model is that occupational hazards may vary systematically as a function of racial composition. I therefore model the occupation-specific intercepts as functions of the racial composition of each occupation, denoted $\pi_{r d}$ for each racial category $r$.

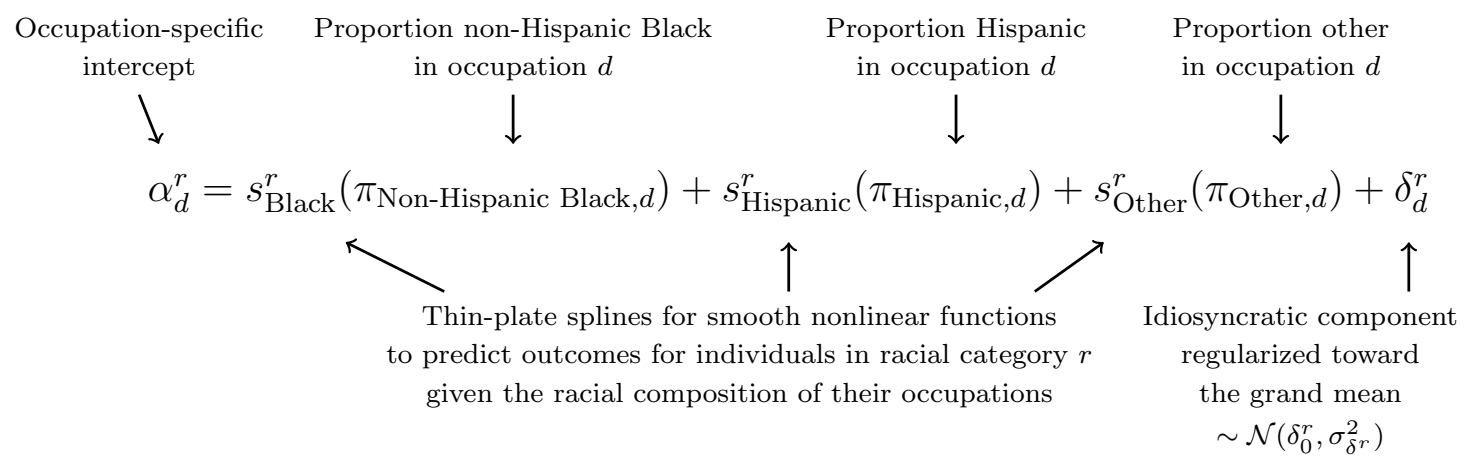

In this specification, the thin-plate splines are regularized toward lines and only the $\delta_{d}^{r}$ component is regularized toward the grand mean. The advantage is that occupations with a given racial composition are all regularized toward each other. Thus, regularization will lead to over-estimation of the outcome in some occupations at a given racial composition and under-estimation the outcome in other occupations with that composition. But the coun- 
terfactual assignment rule will up-weight or down-weight all occupations with a given racial composition in a similar way. As a result, the biases may counterbalance more favorably than in a standard multilevel model specification. It is also important to be mindful that this is entirely a finite-sample consideration: as the sample size with each occupation grows, the idiosyncratic component $\delta_{d}^{r}$ would come to dominate the occupation-specific estimate so that the chosen method of regularization declines in importance.

The only additional hurdle is that the occupation-specific racial compositions $\pi_{r d}$ are not known and must be estimated from the data. I therefore employ a two-step procedure: I first estimate the racial composition of each occupation by random intercept models (Eq. D.3) and then use the estimated $\hat{\pi}_{r d}$ in estimation of the outcome model.

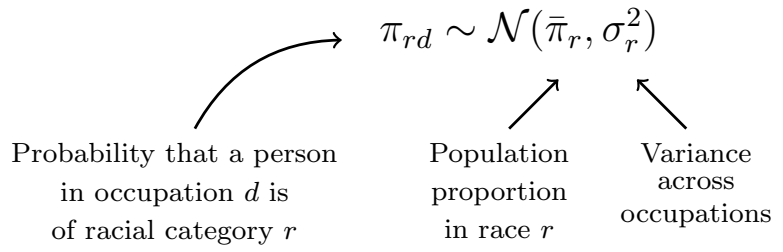

The random intercepts for occupational composition (Eq. D.3) are similar to simply taking the proportion in race $r$ among those observed in occupation $d$. The only difference is that the estimate is regularized toward the population proportion $\bar{\pi}_{r}$ in race $r$. This helps for small occupations, where the racial composition is especially uncertain. The amount of shrinkage $\sigma_{r}^{2}$ is chosen empirically by the mgcv package (Wood, 2017). The analysis captures statistical uncertainty at both steps because uncertainty is estimated by replication methods in which the entire procedure is carried out on repeated samples (Sec. F).

With the estimated prediction function, I then impute the counterfactual probability of work-limiting disability onset $\hat{y}_{i}(d)$ for each person $i$ in each occupation $d$ (Fig 5C). Then, I average over a counterfactual assignment rule for occupations, weighting each potential outcome $\hat{y}_{i}(d)$ by the prevalence of the occupation $d$ among people with the education level of person $i$ (Fig 5E). Finally, I aggregate the person-specific estimates over people in each subpopulation defined by race and ethnicity, using survey weights (Fig 5E). 


\section{D.1 Doubly-robust estimates}

The main text presents models from an approach centered on predicted outcomes. An alternative approach (Fig 16 Panel I) is a doubly robust estimator: build predictions for the probability of assignment to each occupation (analogous to a propensity score) and correct the outcome predictions by their estimated bias (the weighted mean error with inverse probability of treatment weights). Lundberg (2021) discusses this estimator in greater depth. The benefit of a doubly robust estimator is that it is consistent if either the treatment model or the outcome model is correctly specified.

The treatment in the present paper is occupation, with 451 categories. Doubly robust estimators can have high variance when inverse probability of treatment weights are highly variable, which especially occurs when some probabilities of treatment are very near zero (Kang and Schafer, 2007). This means that doubly robust estimation may produce poor estimates for outcomes in extremely rare occupations. To avoid this problem, I focus the doubly robust estimator on a different counterfactual assignment rule: assign occupations from among the 190 largest occupations that collectively contain $90 \%$ of the population of people.

With this simplification, it is still difficult to estimate the factual probability of holding each of the 190 relevant occupations as a function of all observed confounders. Fitting a multinomial logistic regression model is infeasible with so many outcome categories. I therefore break the problem into two parts. I first estimate the probability of holding an occupation in each of the nine major occupational categories in the Census occupational classification system, which are indicated by the first of the three-digit detailed occupation code. Within each major classification, I then estimate the probability of holding each

detailed occupation among the occupations in that major category (between 9 and 33 occupations). The probability of holding an occupation given covariates is the product of the probability of the major category and the probability of the detailed occupation given the major category. I estimate the multinomial models with the multinom function in the nnet 
package in $\mathrm{R}$ (Venables and Ripley, 2002), entering all covariates additively with linear terms for continuous covariates, and with estimation carried out separately for each racial category as in the outcome model specification.

Given the estimated factual probability of treatment, one can learn about the outcomes under the counterfactual assignment rule by weighting each observation by a weight that is the product of the survey weight, the counterfactual probability of being assigned to the factual occupation, and the inverse of the factual probability of the factual occupation (Lundberg, 2021). Using these weights, I de-bias the estimated outcome predictions by subtracting the weighted mean outcome residual from the outcome predictions. Results (Fig 16 Panel I) are substantively similar to the outcome modeling estimates presented in the main text.

\section{E Measurement issues with work-limiting disability in the CPS-ASEC}

There are two important limitations to the operationalization of work-limiting disability in the CPS-ASEC: changes in the survey instrument over time and limitations in the definition of disability for a self report.

\section{E.1 Changes in the CPS-ASEC survey instrument}

Prior to 2014, the CPS-ASEC instrument was relatively constant over time. The household respondent answered a work-limiting disability question for each household member: "(Do you/Does anyone in the household) have a health problem or disability which prevents (you/them) from working or which limits the kind or amount of work (you/they) can do?" Two important changes occurred after that, one in 2014 and one in 2016.

In 2014, the wording of this question remained the same but other aspects of the CPS- 
ASEC underwent a major redesign. ${ }^{8}$ All respondents answered new questions about health insurance, and 3/8 of the sample received income questions in a new format. After 2014, the CPS-ASEC followed the new format for all respondents. Although the work-limiting disability question did not change, Fig 10 Panel A shows that the reported prevalence of work-limiting disability rose substantially under the new questionnaire structure. One reason for this increase might be the change in the order of the questionnaire. In the traditional questionnaire, the module immediately preceding work-limiting disability was about Social Security survivor benefits. In the redesigned questionnaire, the module immediately preceding work-limiting disability was about Supplemental Security Income (SSI), a Social Security program that provides transfers to individuals with disabilities (Czajka et al. 2015:5). Asking someone to report their receipt of SSI may prime them to report a work-limiting disability at a higher rate in the immediately subsequent questionnaire module.

In 2016, the wording of the work-limiting disability question changed to focus explicitly on work-limiting disability in the past calendar year, with an added prompt about limitations that were in place "even for a short time." 9 As illustrated in Figure 10 Panel A, this wording change produced no apparent change in the proportion reporting a work-limiting disability.

Despite both of these changes, the Black-white disparity in work-limiting disability remains remarkably consistent across the years 2005-2020 (Fig 10 Panel B). The main analyses therefore pool over all years. Fig 16 Panel G shows that the results are similar if subsetted to the years preceding either change, and Panel $\mathrm{H}$ shows a similar result if subsetted to the 2014-2020 period in which one or both changes were in effect.

\section{E.2 Validity in the meaning of the disability question}

Self-reported work-limiting disability faces the same problems of survey measurement that affect any question: it captures disabilities that limit work only to the extent that respondents

\footnotetext{
${ }^{8}$ Details of the CPS-ASEC 2014 redesign are available in a BLS memo: https://cps.ipums.org/cps/ resources/other_docs/ASEC_Redesign.pdf

${ }^{9}$ Full question wordings are available at https://cps.ipums.org/cps-action/variables/DISABWRK\# questionnaire_text_section.
} 
A) Reports of a work-limiting disability increased when the questionnaire changed

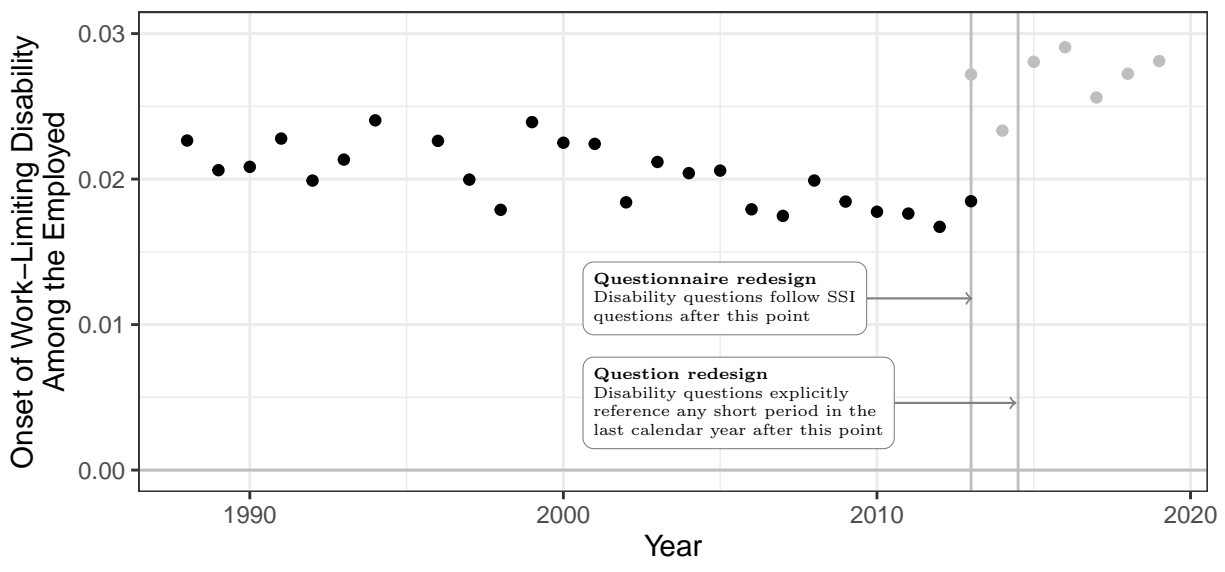

B) The key disparity of interest remained roughly constant

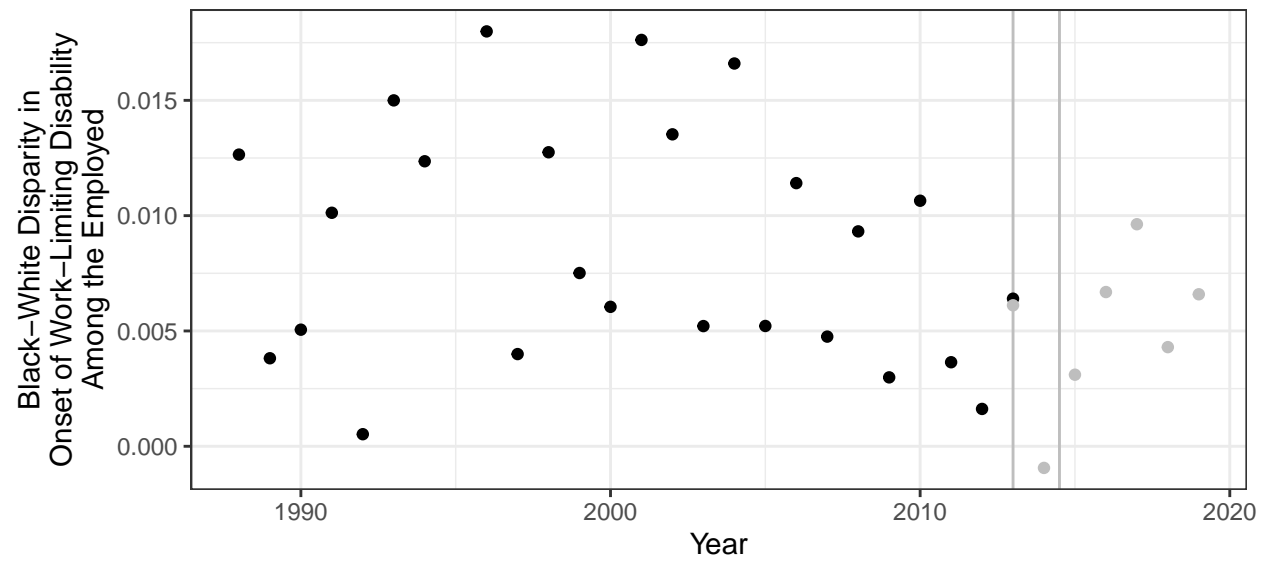

Fig. 10. Changes to the survey instrument affected levels but not disparities in reported work-limiting disability. For consistency with other plots, the $x$-axis here refers to the first year of observation, but the outcome is measured in the second year of observation. Therefore, CPS questionnaire changes in 2014 and before 2016 correspond to vertical lines placed at 2013 and before 2015 in this plot. In 2014, the CPS questionnaire was redesigned and modules were re-ordered so that the disability questions immediately followed the Supplemental Security Income questions instead of the Survivor Benefit questions. The redesigned questionnaire was given to $3 / 8$ of the sample (gray dot on the vertical line), while $5 / 8$ of the sample received the original questionnaire (black dot on the vertical line). Before 2014, everyone answered the traditional questionnaire (black dots) and after 2014 everyone answered the redesigned questionnaire (gray dots). Beginning in 2016, the question about "a health problem or a disability which prevents him/her from working or which limits the kind or amount of work" was changed to reference the past calendar year and to include the prompt for conditions lasting "even for a short time." The 2014 redesign increased reported disability, while the 2016 question change appears to have had little effect on reported disability. The Black-white disparity in disability onset is roughly constant over both changes to the survey instrument. The main analyses therefore pool over both formats of the question, with alternative specifications separating at the more consequential update (2014) reported in Fig 16 Panels G and H. 
understand them as such. Hale (2001) critiques the CPS work-limiting disability question for defining disabilities in terms of work limitations rather than in terms of specific medical impairments. In an evaluation that compares the CPS work-limiting disability question to a broader definition of disability from a series of questions in the National Health Interview Survey, Burkhauser et al. (2002) show that the work-limiting disability question estimates a smaller population with a disability but that employment trends in both populations are similar. Maestas et al. (2019) collect new data to show that those who report a workplace accomodation for health reasons may still answer "no" to a question about whether they have a work-limiting disability. One should therefore understand a work-limiting disability as one particular operationalization of a broader concept of disability. It is possible that selfperceptions of disability deviate from how a medical professional would diagnose a disability, and those deviations may differ across individuals. Yet, one's own perception that one has a work-limiting disability may be a worthy object of study in its own right; it is real for the person involved even if it is distinct from the diagnosis a medical professional would provide. A further advantage of accepting these self-reports as worthy of study is the ability to use the very large samples of the CPS with detailed occupational information linked over a 12-month interval.

\section{F Statistical inference by replicate weights}

Statistical inference (e.g. standard errors and confidence intervals) involves a claim about how an estimator would vary if applied to hypothetical new samples drawn from the sampling frame by the same procedure used to generate the observed sample. Standard procedures assume a simple random sample and apply only if that is how the data were generated. The CPS is not a simple random sample. Instead, geographic Primary Sampling Units (PSUs) are selected within geographic strata, and then geographic Ultimate Sampling Units (USUs, clusters of households) are selected within PSUs by systematic sampling after sorting 
USUs systematically by labor market characteristics. Further, selection occurs with unequal probabilities in order to yield accurate estimates in subpopulations (e.g. state-specific unemployment estimates). This complex multistage cluster design renders standard inference procedures inappropriate. Further, as the analyst I cannot directly observe the strata, PSU, or USUs because these geographic identifiers are hidden in the data files for privacy reasons.

Inference therefore relies on a set of replicate weights constructed by the Bureau of Labor Statistics. Fig 11 summarizes the sampling process of the CPS and the procedure by which the BLS constructs the replicate weights (Bureau of Labor Statistics $2006 \mathrm{Ch}$. 14, Fay and Train 1995). Fig 12 illustrates the mechanical process by which the replicate weights yield a variance estimate: by conducting the estimation algorithm with each set of replicate weights in turn and estimating variation across the sets of replicate weights, one can simulate how the estimator would vary across repeated samples drawn from the CPS design. Because this entire procedure is computational, it is possible to estimate sampling variability by replicate weights even for potentially complicated estimation algorithms that involve regression, prediction, and aggregation to counterfactual summaries.

An advantage of the replicate weight procedure is that it applies equally well to any estimation algorithm, even one that is complex. Within each replicate, I estimate the predictive model for the outcome, impute unobserved counterfactuals, take averages, and report an estimate. The confidence intervals may not have proper coverage because the estimator is slightly biased due to regularization. However, they do capture how the estimator would vary across repeated samples drawn by the sampling design of the CPS. 
Sampling Process

Begin with the sampling frame:

All residential addresses in the U.S.
Replicate Process

Begin with the sample:

Chosen households
Define geographic Primary

Sampling Units (PSUs) within

two types of geographic strata.

Non-Self-Representing Strata (NSR):
Each small-population PSUs is

placed in a stratum with others.

Self-Representing Strata (SR):

Each large-population PSU is

placed in a stratum alone.

Select Ultimate Sampling Units (USUs)

(clusters of households) in each PSU
PSUs and strata are already defined.

For privacy reasons, these steps are conducted by the Bureau of Labor Statistics and are not visible to researchers.

Interview those households Estimate $\hat{\theta}$ with weights $\vec{w}$

The variance of an estimator is how it would vary across repeated CPS samples drawn this way
Analyze those households

Estimate $\hat{\theta}_{r}^{*}$ with each replicate weight $\vec{w}_{r}$

The estimated variance is a function of variation across the 160 replicates:

$$
\hat{\mathrm{V}}(\hat{\theta})=\underset{\uparrow}{\frac{4}{160}} \sum_{r=1}^{160}\left(\hat{\theta}_{r}^{*}-\hat{\theta}\right)^{2}
$$

Note: The factor of 4 is needed because the replicates inflate and deflate weights without completely zeroing out any weights.

Fig. 11. Replicate weights mimic repetitions of the CPS sampling process. Illustration is based on the BLS description (Bureau of Labor Statistics, 2006; Fay and Train, 1995). Because the CPS is not a simple random sample, standard approaches to statistical inference are inappropriate. Replicate weights simulate variation that comes from selection of geographic Primary Sampling Units (PSUs) within strata. For PSUs selected with probability one, replicate weights mimic variability within strata. Although the weight construction procedure is described by the BLS, as the researcher I only have access to the generated weights $\vec{w}$ and replicate weights $\left\{\vec{w}_{r}\right\}_{r=1}^{160}$. Inference in this paper proceeds by conducting the entire estimation algorithm repeatedly with each set of replicate weights, calculating the variance of the point estimate, and constructing a confidence interval by a normal approximation. 
Point estimate

Define weight to be

the main survey weight
Replicate estimates

Define weight to be one of 160 sets of replicate weights
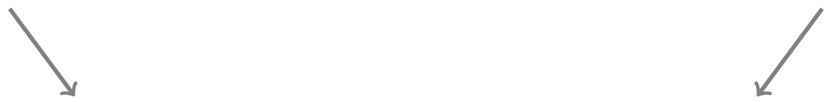

\section{Estimation Algorithm}

Fit a Generalized Additive Model weighted by weight for onset of a work-limiting disability as a function of covariates and occupation

Predict the outcome each person would expect if assigned an occupation by the counterfactual assignment rule

Aggregate across people using weight

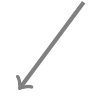

Produces a point estimate $\hat{\theta}$
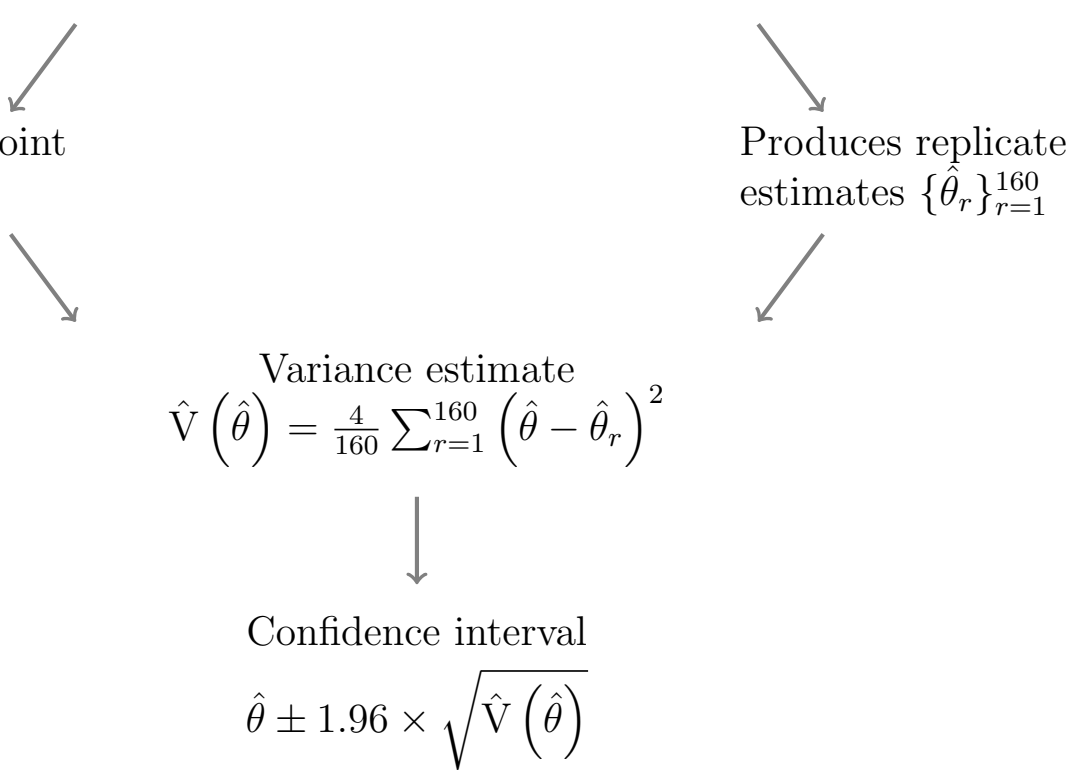

Fig. 12. Procedure to construct confidence intervals with replicate weights. The entire estimation procedure - regression, imputation, and aggregation - can be placed within an algorithm that accepts a weight as the input and returns an estimate. Passing the main survey weight through that algorithm yields a point estimate. Passing the replicate weights through the algorithm yields a set of 160 replicate estimates. Pooling across these estimates yields an estimated variance. By a normal approximation, the point estimate and estimated variance produce a $95 \%$ confidence interval. 


\section{G Supplemental Figures}

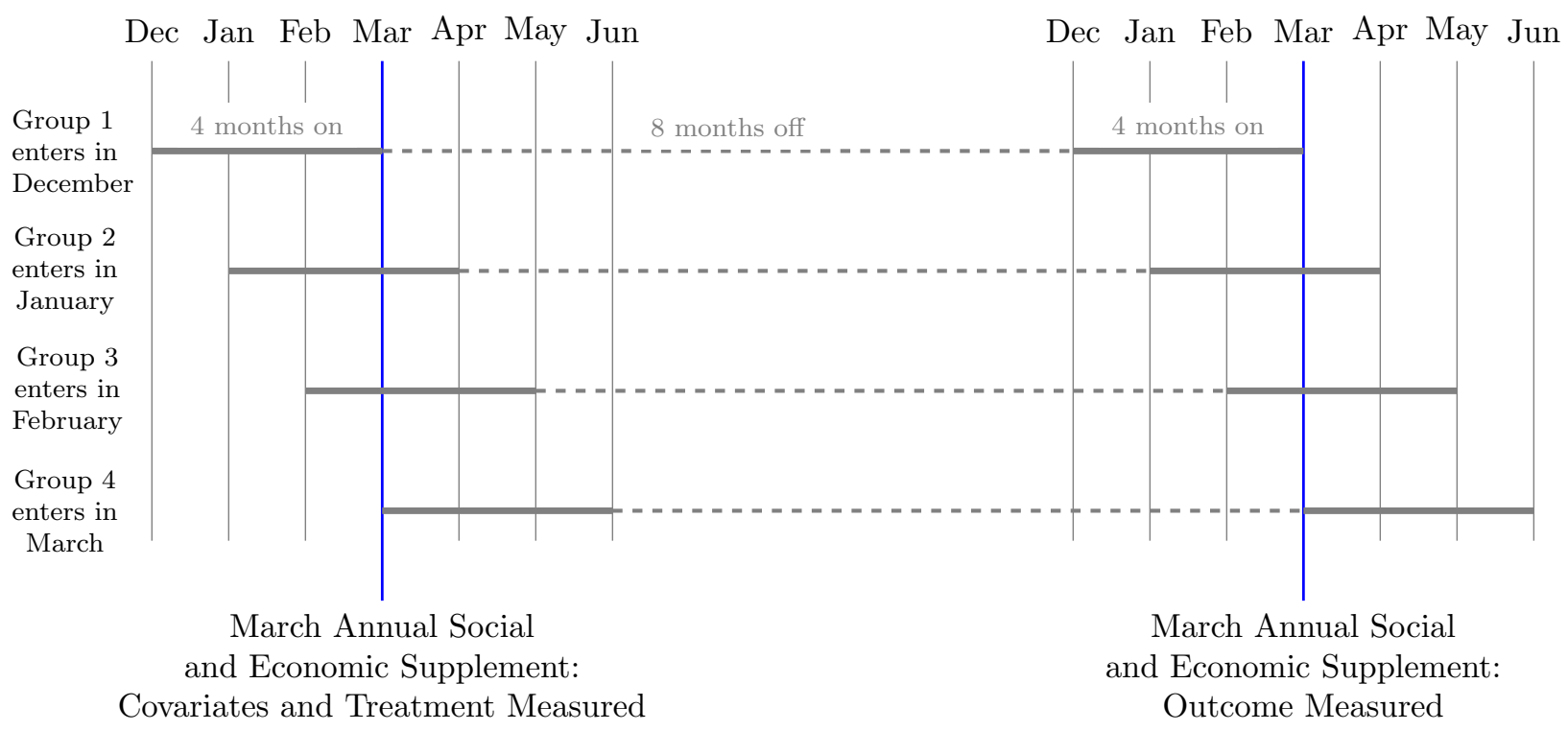

Fig. 13. Panel structure of the Current Population Survey. Households are sampled and assigned to rotation groups, which enter the panel on different months throughout the year. Each rotation group is included in the monthly survey for four months, excluded for eight months, and then included again for four more months. A basic survey is administered each month with the primary goal of tracking the unemployment rate. The Annual Social and Economic Supplement is a large survey administered each March. Because of the panel structure, households that enter the CPS panel in December to March are included in the ASEC survey for two adjacent years. The ASEC also includes oversamples which follow a different panel structure and are not linkable across years. For example, since 2001 the ASEC has included oversamples of low-income households drawn from those who enter the CPS in other months, with the goal of providing estimates relevant to evaluations of the State Children's Health Insurance Program. Because the oversamples are not linkable across years, they are excluded from the linked data analyses in this paper. See Flood and Pacas (2017) for more information. 


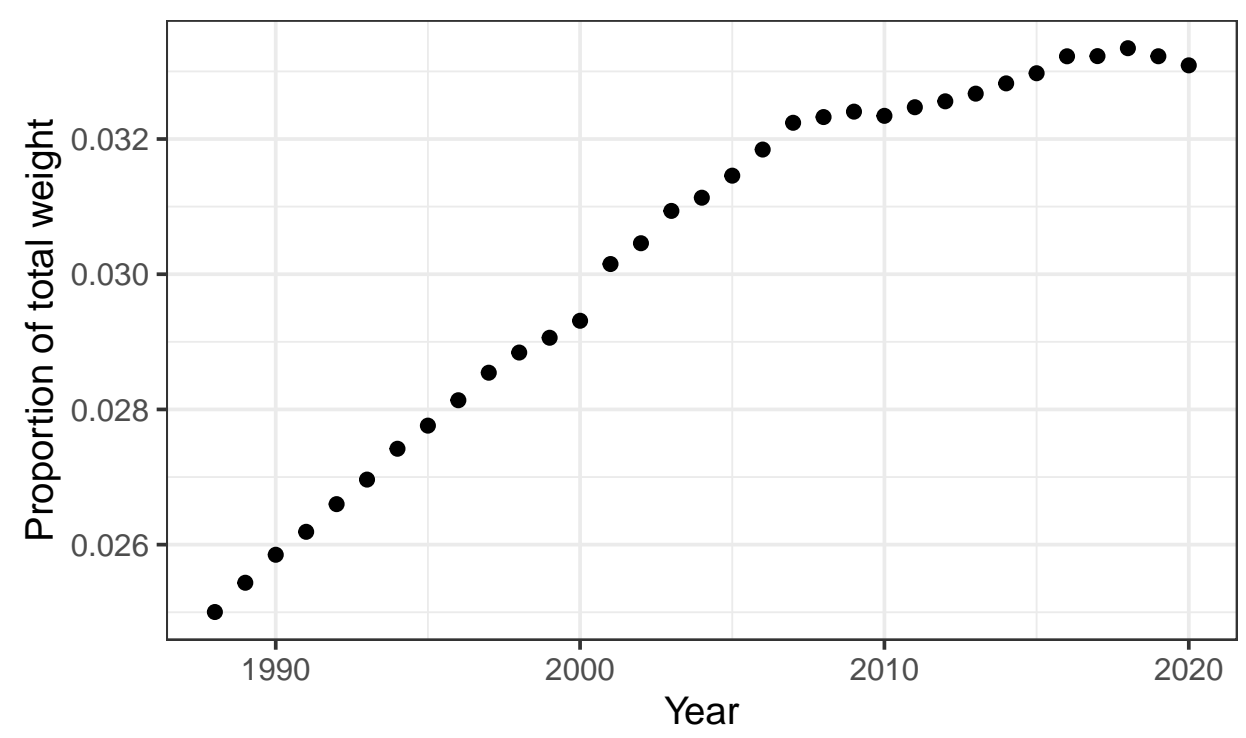

Fig. 14. The total weight on the sample increases over time. This is because the U.S. population ages 25-60 increases in size over time; when drawing inference about this population, the more recent years are weighed more heavily because they contain a larger share of the total population.

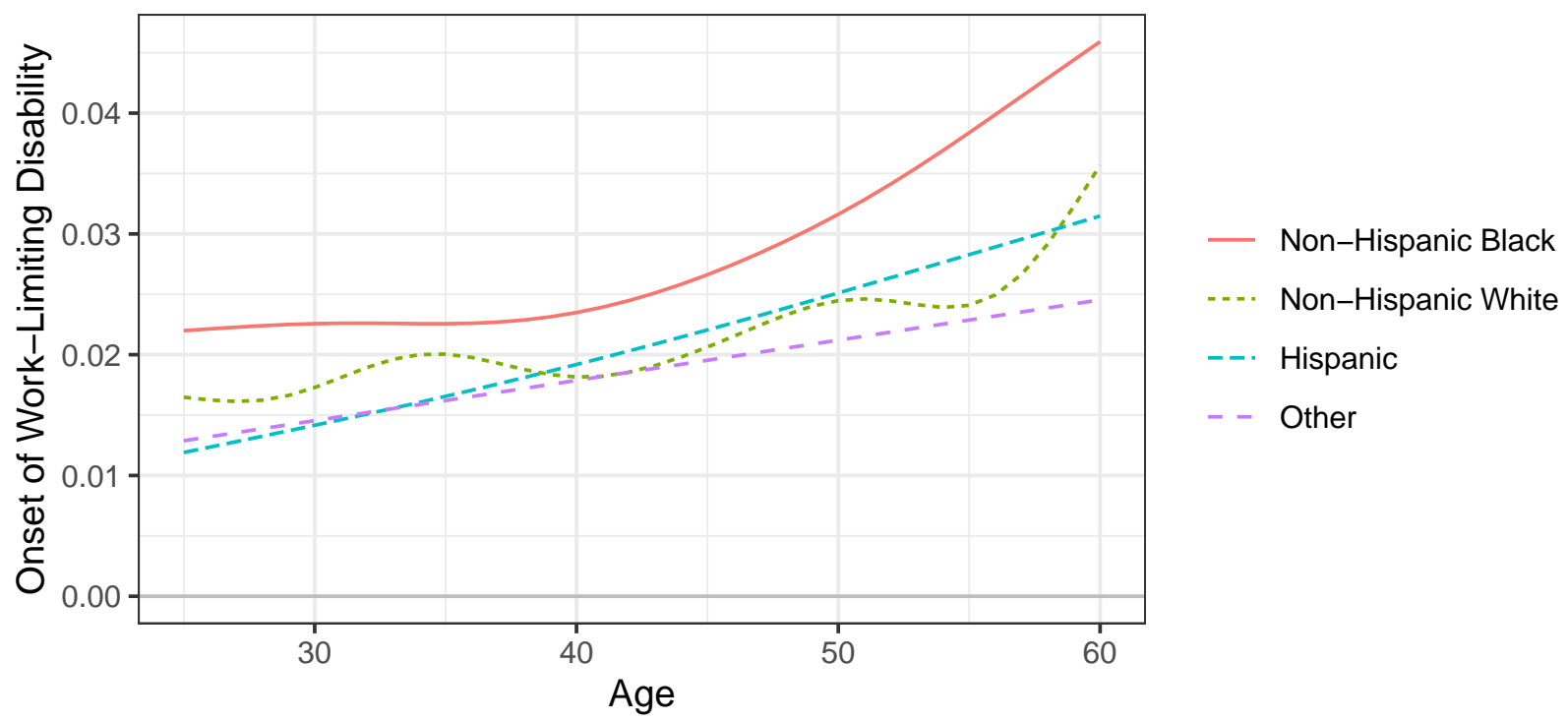

Fig. 15. Conditional association between age and work-limiting disability. These smooth terms are visualized here to build intuition for the Generalized Additive Model (GAM, Wood 2017) predicting the onset of a work-limiting disability as a function of all predictors. The association is free to differ by race because models are estimated separately by race, and the smooth curve is not restricted to any parametric form (e.g. quadratic). These curves have no causal interpretation; they depict the population average conditional association between age and the outcome. 

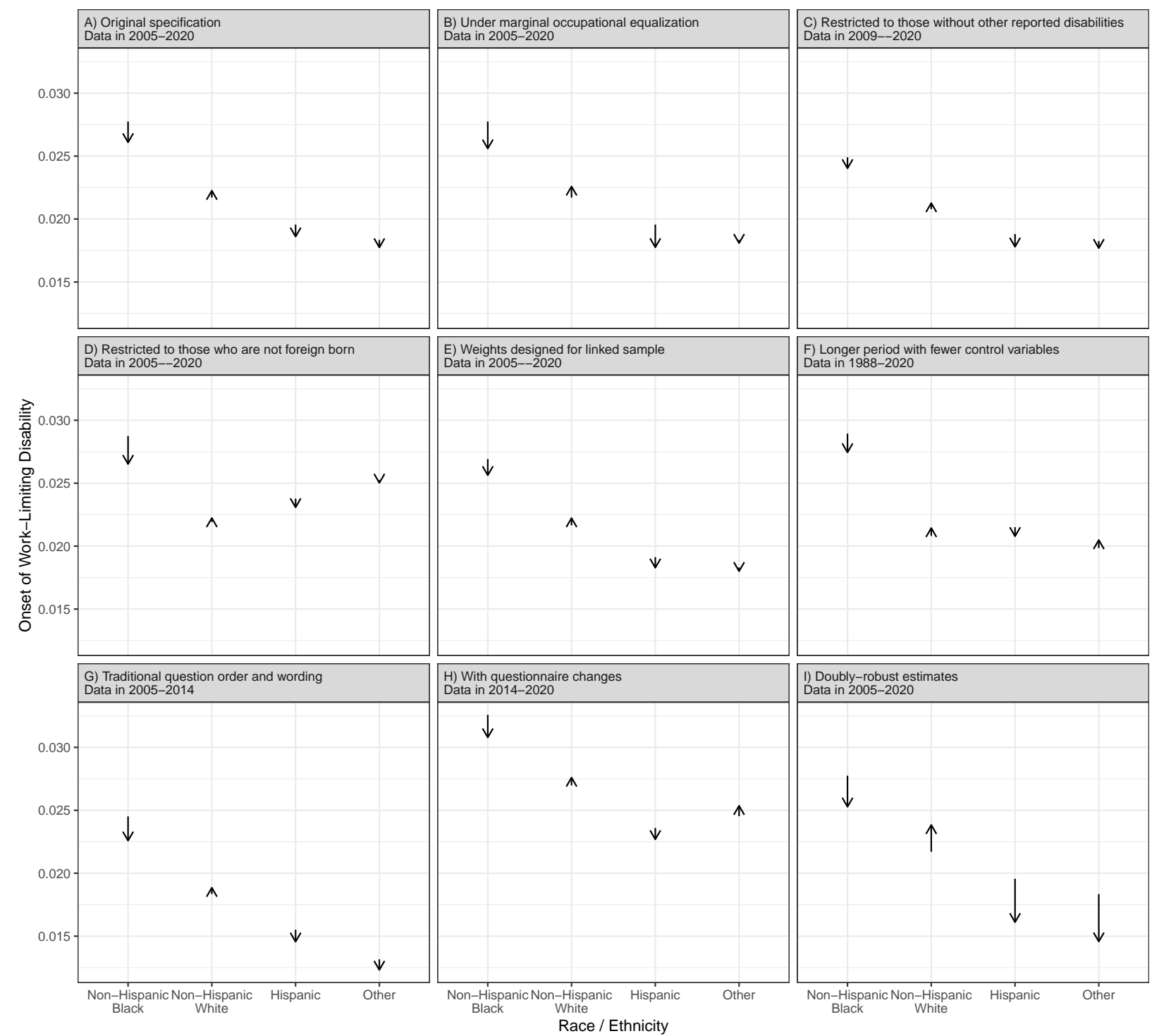

Fig. 16. Alternative specifications for the main result. Each arrow goes from the factual prevalence of work-limiting disability to the counterfactual prevalence under equalization of occupation assignments within education. In each panel, the reported years correspond to year $t$ in which covariates and occupation are measured; the onset of a work-limiting disbaility is in the following year $t+1$. Panel A is the specification from the main text $(N=247,847)$. Panel B shows results for marginal equalization: assign occupations by their population prevalence for all individuals regardless of education. Panel $\mathrm{C}$ uses additional data available in 2009-2020 to subset to those who report never leaving a job for health reasons and who report no difficulty with hearing, vision, remembering, walking or climbing stairs, performing basic activities outside the home along, or taking care of personal needs $(N=165,961)$. Panel D subsets to those who are not foreign-born $(N=208,256)$. Panel E uses the LNKFW1YWT weights provided by IPUMS for the subsample that links across years instead of the standard ASECWT weight $(N=247,847)$, which cannot be used in the main analyses because replicate versions of that weight are not available. Panel $\mathrm{F}$ provides point estimates for the full 1988-2019 period in which work-limiting disability was measured by the CPS, at the cost of not adjusting for foreign-born status or lagged self-rated health $(N=531,896)$. Panel $\mathrm{G}$ subsets to those who received the original version of the disability question in both year $t$ and year $t+1(N=156,247)$. Panel $\mathrm{H}$ subsets to those who received the new version of the disability question in both year $t$ and year $t+1$, where the new version additionally prompted for disabilities lasting a short time $(N=71,351)$. Panel I shows results with a doubly-robust estimator for a slightly modified assignment rule (see Appendix D.1). 